\title{
Shielding NSLS-II Light Source: Importance of Geometry for Calculating Radiation Levels from Beam Losses
}

\author{
S.L. Kramer \\ Operations and Accelerator Design Consulting \\ 568 Wintergreen $\mathrm{Ct}$ \\ Ridge, NY 11961 \\ and \\ V. J. Ghosh, M. Breitfeller, and W. Wahl \\ NSLS-II, Brookhaven National Laboratory ${ }^{1}$ \\ Upton, NY 11973 \\ August 6, 2016
}

Abstract

Third generation high brightness light sources are designed to have low emittance and high current beams, which contribute to higher beam loss rates that will be compensated by Top-Off injection. Shielding for these higher loss rates will be critical to protect the projected higher occupancy factors for the users. Top-Off injection requires a full energy injector, which will demand greater consideration of the potential abnormal beam miss-steering and localized losses that could occur. The high energy electron injection beam produces significantly higher neutron component dose to the experimental floor than a lower energy beam injection and ramped operations. Minimizing this dose will require adequate knowledge of where the miss-steered beam can occur and sufficient EM shielding close to the loss point, in order to attenuate the energy of the particles in the EM shower below the neutron production threshold $(<10 \mathrm{MeV})$, which will spread the incident energy on the bulk shield walls and thereby the dose penetrating the shield walls. Designing supplemental shielding near the loss point using the analytic shielding model is shown to be inadequate because of its lack of geometry specification for the EM shower process. To predict the dose rates outside the tunnel requires detailed description of the geometry and materials that the beam losses will encounter inside the tunnel. Modern radiation shielding Monte-Carlo codes, like FLUKA, can handle this geometric description of the radiation transport process in sufficient detail, allowing accurate predictions of the dose rates expected and the ability to show weaknesses in the design before a high radiation incident occurs. The effort required to adequately define the accelerator geometry for these codes has been greatly reduced with the implementation of the graphical interface of FLAIR to FLUKA. This made the effective shielding process for NSLS-II quite accurate and reliable. The principles used to provide supplemental shielding to the NSLS-II accelerators and the lessons learned from this process are presented.

\footnotetext{
${ }^{1}$ This work is supported in part by the U.S. Department of Energy (DOE) under contract No. DE-AC02-98CH1-886.
} 


\section{Introduction}

The NSLS-II synchrotron light source (SLS) was designed as an Ultra-high brightness X-ray SLS with $3 \mathrm{GeV}$ energy, sub-nm beam emittance, high beam current and short bunch electron beam in a $792 \mathrm{~m}$ circumference ring. The novel ring design will naturally lower the beam emittance as damping wigglers and other strong undulators are added to the ring [1]. With these parameters the beam lifetime is expected to be small $(\sim 3 \mathrm{~h}$ at $500 \mathrm{~mA})$ requiring Top-Off injection into the ring to stabilize the beam power heating to the beamline optics and accelerator components. The required injected charge rate is $\sim 7.5 \mathrm{nC} / \mathrm{min}$ under these circumstances. Although oversight committees are quite concerned about miss-steering the injection beam down an open beam line shutter during TopOff, this is highly unlikely. With Top-Off requireing full energy injection, the more likely abnormal event is miss-steering the injection beam with the beam hitting components inside the storage ring tunnel. Because of the intense forward cone of the EM shower, this requires the installion of supplemental shields (SS) at the most likely loss locations to attenuate this shower before hitting the outer shield walls.

The failure to identify a possible miss-steered beam location and the intense forward radiation exposure the EM shower can create, was observed early in the commissioning of the NSLS-II linac, which had a major impact on the commissioning. The bulk shielding around the linac tunnel was designed only to address transverse radiation from a relatively low fractional beam loss and not the intense forward radiation shower from the entire beam current. Even if commissioning took place at lower beam currents, future operation would require full beam intensity from the injector and miss-steering can and are likely to occur due to equipment failure and operational errors. Those are the times when occupancy resitrictions won't easily be implemented due to increased user activity to implement and improve their beam lines. The requirement to keep the dose to staff and users "As Low As Reasonably Achievable" (ALARA) means effort needs to be spent understanding where these possible loss locations are and a reliable estimate of the dose each fault condition could create to occupied areas.

\section{Bulk Shield Wall Specifications (Analytic Shielding Model Estimates)}

The bulk shield walls for NSLS-II were designed assuming a specified fraction of the beam current, $\mathrm{I}_{\mathrm{av}}$, was lost at any location along the accelerator (Linac, booster or storage ring) vacuum chamber at the operational electron beam energy, E, [2]. The concrete wall thickness was increased until the calculated dose equivalent rates outside the shield walls were less than $0.5 \mathrm{mrem} / \mathrm{h}$ ( 5 $\mu \mathrm{Sv} / \mathrm{h}$ ). The dose rate was calculated using an Analytic Shielding Model [ASM] that assumes the radiation of concern originates from a beam having a beam power, J, hitting a thick target [2]. The thick target needs to have greater than 10 radiation lengths and greater than 5 Moliere radii in transverse size, in order to generate a significant EM shower and transfer sufficient energy to the shower particles. All radiation components are assumed generated in this target with source terms for each components of radiation emitted (i. e. gamma rays, low energy and high energy neutrons of concern here) expressed as dose equivalent factors $F_{i}$ (source term) for each component $i$. These $F_{i}$ are the unshielded ambient dose equivalent (or dose rate) for that component per unit of incident beam energy $\mathbf{J}$ (or power), at a distance $\mathrm{R}=1 \mathrm{~m}$ from the target. Each radiation component is then shielded by the material of thickness $t$, with an average attenuation length $\lambda_{i}$ for the component, $i$. The shielded total dose equivalent rate $\mathrm{H}[\mu \mathrm{rem} / \mathrm{s}, 0.01 \mu \mathrm{Sv} / \mathrm{s}]$ is estimated by the sum of each attenuated radiation component for the incident total beam power loss, $\mathrm{J}=\mathrm{E} / \mathrm{e} * \mathrm{I}_{\mathrm{av}}(\mathrm{J} / \mathrm{s})$, at a total distance $\mathrm{R}$ from the target, by the equation

$$
\mathrm{H}=\left(\mathrm{J} / \mathrm{R}^{2}\right) \cdot \Sigma_{\mathrm{i}} \mathrm{F}_{\mathrm{i}} \cdot \exp \left[-\mathrm{t} / \lambda_{\mathrm{i}}\right]
$$


This equation is only strictly valid for transverse radiation dose rate at $\sim 90^{\circ}$ (transverse tunnel walls) to the incident beam direction and for thick targets as described above. Typically the bulk shield walls will be transverse to the beam direction, however in light sources a forward ratchet wall is provided for the photon beam transport out of the tunnel. In the forward direction the ASM can still be used but with larger values for the $\mathrm{F}_{\mathrm{i}}$, that increase proportionally to the incident particle beam energy, E. The values for $F_{i}$ used to design NSLS-II accelerator shield walls $[2,3]$ are listed in Table I. The neutron component is actually broken into two components; a low energy neutron ( $E_{n}<25 \mathrm{MeV}$ ) and a high energy neutron ( $\mathrm{En}>25 \mathrm{MeV}$ ) terms, but only their total is listed in the table. The shield wall thickness was adjusted until the ASM estimated dose rate given by Eq.1. (for a specified operational beam power loss $\mathrm{J}$ and distance $\mathrm{R}$ from the accelerator vacuum chamber) was below the targeted value, typically less than $0.5 \mathrm{mrem} / \mathrm{h}(5 \mu \mathrm{Sv} / \mathrm{h})$ for the experimental floor of the NSLS-II Storage Ring (SR) and other occupied areas.

The Monte Carlo radiation transport code FLUKA [5] was used to estimate the dose equivalent factors for electron beams hitting a $30 \mathrm{~cm}$ long x $10 \mathrm{~cm}$ diameter iron target. The dose distribution around the target for two beam energies is shown in Figure 1. This shows the increased forward dose rate as the beam energy increases, but the transverse dose shows less energy dependence. The dose equivalent, at $\mathrm{R}=1 \mathrm{~m}$ from the unshielded target was calculated for electron energies $(0.2,1$, and $3 \mathrm{GeV})$. Table I lists the $3 \mathrm{GeV}$ normalized dose equivalent values calculated with FLUKA at $1 \mathrm{~m}$ from the target and scaled by the beam energy in Joules. These values can be compared with the $F_{i}$ values used in the ASM [2]. The calculated FLUKA Amb74 dose equivalent values use the fluence-to-ambient dose equivalent conversion factors proposed by the ICRP Publication 74 [6]. The Amb74 dose is the ambient dose equivalent (Quality Factor weighted) for the radiation field penetrating a $1 \mathrm{~cm}$ depth in the direction of an oriented and expanded radiation field for an ICRU sphere [6]. All references to calculated dose in this paper will refer to ambient dose equivalent. The dose values listed in Table I were scored for all particles (Total Dose), the gamma ray and the total neutron dose components of the radiation field. The difference between the total dose and the sum of the other two dose components yields the charged particle component, which isn't included in the ASM, but is always present where gamma and neutrons are part of the radiation field. Also listed is the exponential factor from a power law fit to the dose as a function electron beam energy E, for the three energies simulated. For the transverse dose calculated with FLUKA, the scaling with $\mathrm{J}$ of the ASM the $\mathrm{F}_{\mathrm{i}}$ appears quite reasonable, since the power of $\mathrm{E}$ dependence parameters being compatible with zero. However the FLUKA data does show a small dependence on $\mathrm{E}$ for the neutron component. This arises from a greater number of photons being produced in the target with energies above the neutron production threshold of $\mathrm{E}>2 \mathrm{MeV}$ as $\mathrm{E}$ increases. Clearly the ASM over estimates the total and gamma dose values for these source terms ( 2.5 times the gamma dose compared to Amb74 dose), however the neutron dose is under estimated and fails to include this increasing source term with E. This dependence is shown in Figure 2 where the FLUKA simulated transverse Amb74 doses at a distance of 1 meter from the target are plotted versus longitudinal coordinate for $\mathrm{E}=0.2,1$ and $3 \mathrm{GeV}$. The total (and gamma) dose data shows an increase of greater than $20 \%$ at angles relative to beam direction $\theta<70^{\circ}(\mathrm{z}>40$ $\mathrm{cm})$, as compared to the $\theta=90^{\circ}$ dose $(\mathrm{z}=0)$. The neutron dose, Fig.2(b), shows the largest energy increase from 0.2 to $1 \mathrm{GeV}$ and a smaller from 1 to $3 \mathrm{GeV}$, as well as an isotropic production distribution around the center of the target $(\mathrm{z}=0)$.

The FLUKA simulated forward ambient dose equivalent were calculated for a cylindrical detector (dose scoring region) at $\mathrm{R}=1 \mathrm{~m}$ from the target and are normalized by the beam energy in Joules. The normalized ambient dose equivalent $\left(\mu \mathrm{rem} \cdot \mathrm{m}^{2} / \mathrm{J}\right.$ or $0.01 \mu \mathrm{Sv} \cdot \mathrm{m}^{2} / \mathrm{J}$ ) is plotted for radial distance from the beam axis in Figure 3 for the total and neutron components at 0.2, 1, and 3 $\mathrm{GeV}$. These shows the increased energy dependence of the source terms at small angles less than 
$10^{\circ}$. The $3 \mathrm{GeV}$ data show the dose is more peaked in the forward direction resulting from the EM shower penetration of the target. In addition, Table I also shows that the neutron component also has an additional energy scaling in the forward direction that is greater than the transverse component.

Although the transverse $\mathrm{F}_{i}$ used by the ASM have a larger gamma source term, the neutron component $F_{i}$ is lower than the FLUKA estimates. The forward dose rates are significantly higher for the gamma term and penetration of the EM shower from the target will also contribute to distant neutron production in accelerator and shielding material that will not have the $\mathrm{R}^{-2}$ dependence that the target produced neutrons will have. This secondary production of neutrons will have an important contribution for shielding the SR for the higher injection energy. Figure 3 shows the $0^{\circ}$ gamma component has an energy dependence that's almost linear in E, while it is nearly energy independent at larger radii (angles) from the beam axis. The neutron component shows a similar energy increase at almost all radii (angles) but its dependence is closer to square root of the beam energy. This is the result of increased fluence of the higher energy gamma rays interacting in the target material.

When the high energy EM radiation off the target interacts with shielding material, e.g. a shield wall, it will introduce a secondary source point for neutron production which will not have the $\mathrm{R}^{-2}$ dependence from the target distance. This is shown in Figure 4 for the shielded $(t=1 \mathrm{~m}$ concrete) dose equivalent factors for a dose measurement point $\mathrm{R}=2 \mathrm{~m}$ from the target, for different shield locations relative to the target (near $10 \mathrm{~cm}$ or far $1 \mathrm{~m}$ ). The gamma ray component shows little energy dependence when the shield is near the target, since the solid angle changes little for the fixed dose measurement, while when the shield is closer to the dose scoring location there is a small increase with the higher beam energy. The neutron component shows greater energy dependence for the shield near to the target since the gamma or electron solid angle to the shield is larger yielding greater neutron production in the shield (isotropic source). When the shield is far from the target there still is significant dependence on the beam energy but not as great due to the smaller solid angle from the target for the gamma generation source. The ASM doesn't take this secondary particle production into account and since its estimates don't depend on where the shield is placed relative to the dose measurement location. The ASM estimated ambient dose equivalent values for this transverse shielded radiation are listed in Table II, along with the FLUKA estimates that are shown in Figure 4.

The ASM shielding estimates, since they use dose equivalent factors values greater than those calculated by FLUKA, will overestimate the dose rates and therefore the shielding material thickness needed to meet the targeted dose level. This might be considered to be a conservative approach. However since the ASM overestimates electromagnetic component levels to the total dose as compared to neutron levels, the use of this model to provide supplemental shielding, could result in choosing shielding material which is less efficient in reducing the neutron component of the dose. Despite this the ASM has been extended to include additional shielding materials, $\mathrm{j}$, along the path length between the target and the dose measurement point by including the product of the attenuation for the thickness of material, $\mathrm{t}_{\mathrm{j}}$, with the attenuation lengths, $\lambda_{\mathrm{ji}}$

$$
\mathrm{H}=\left(\mathrm{J} / \mathrm{R}^{2}\right) \cdot \Sigma_{\mathrm{i}} \mathrm{F}_{\mathrm{i}} \cdot \Pi_{\mathrm{j}} \exp \left[-\mathrm{t}_{\mathrm{j}} / \lambda_{\mathrm{ji}}\right]
$$

This equation does not include the length nor width of the shields provided and therefore doesn't determine whether to use the constant $F_{i}$ of the transverse direction or the linear $E$ dependent $F_{i}$ in the forward direction. This lack of geometrical consideration of the ASM will cause difficulty is estimating the path length of material and source terms. These uncertainties could easily exceed the assumed conservative ASM estimated levels outside the shield wall, especially when abnormal beam losses hitting thin targets occur in the forward direction. As a result of this uncertainty NSLS- 
II local shielding design effort used estimates of the dose levels outside the shield wall calculated by FLUKA for the detailed geometry.

\section{Shielding for Abnormal Beam Losses}

The ASM Eq. (1) was used to define the outer shield wall thickness based on an assumed operational beam loss value around the SR [2]. However as shown above the forward EM shower has large source terms with a narrow angular distribution that could greatly exceed the shield wall attenuation when beam is lost from the accelerator. To address these events supplemental (local) shielding will be provided that will intercept the miss-steered beam and attenuate and scatter the beam energy before it hits the shield walls. The Local Shielding Design Coordinating Group (LSDCG) was formed to identify these potential beam loss locations and to design and install these local shields where needed. The LSDCG included representatives from accelerator physics, radiation physics, mechanical and electrical groups. The basic operating principal was to fully understand and document the electrical and mechanical limits of the installed accelerator components and range of beam energy and current possible from each accelerator [7]. These limits included the full range of possible fields for all magnets limited, only be their power supply maximum output capability or limited by a credited Personnel Protection System (PPS) interlock. The full range of beam energies that could be delivered (not just assumed range of operations) was also considered, limited only by the limits of the accelerator providing the beam or by a PPS interlock. With these parameters available each magnetic element was studied for the possible misssteering angles and positions exiting the element for beam anywhere inside the vacuum chamber ahead of that element. The ensemble of these output angles was collected and the maximum and minimum angles determined. All magnets; dipoles, quadrupoles, sextupoles, steering and kicker magnets were studied for their normal field components. No assumption of shorted poles changing the multipole field components was made. The only other condition imposed was a credited control that the major bending dipoles had their correct bending polarities (not required for kickers or correctors). Since all dipole polarities were assured to be correct (the magnets were tested at the operational field using a formal procedure with formal documentation of the results which have been inspected in the accelerator readiness review), all dipoles that are powered in series are measured to have the same field and polarity values as the first series magnet in the beam transport. This ensures that only the first dipole could have a zero bend angle issue for radiation risk. Figure 5 shows the collection of possible steering positions and angles out of the $6^{\circ}$ SR dipole for any possible beam or magnet parameter (within these limits) and beam positions inside the vacuum chamber ahead of the dipole (not the first dipole which could have zero field) [7]. This included all possible powering of the quadrupoles, sextupoles and correctors ahead of the dipole, which were not constrained to their design value or polarity. Beam could exit this dipole through the photon aperture (shown in Fig. 5), but not with the same angle as photons from upstream insertion devices (IDs). Similarly beam could have the same angle as the photon beam but they would exit the dipole at a position that would be intercepted by absorbers or collimators downstream of the dipole. These output beam angles and positions and all in between values were then projected to the tunnel shield walls and the path length of material they intercepted (magnet yokes or local shields) determined. The radiation risk to the area outside the shield wall was then determined and if material was insufficient to attenuate the EM shower to meet the NSLS-II Shielding Policy (NSP) level, a supplemental shield (SS) was designed to absorb and spread the EM shower (from vacuum pipes or thin magnet yokes) from hitting the shield wall. These SSs were designed as close as possible to the beam loss point to reduce their volume (cost) and increase their effectiveness. The accelerator geometry (shield walls, major magnets, vacuum chambers, magnet support girders and the SS's) was modeled with FLUKA and the dose rate simulated to the areas of concern outside the shield walls. The SSs designs were modified as needed to reduce this dose rate from these abnormal beam 
losses to meet the NSP [8]. In most cases the SS were made of lead but in some locations far from magnetic fields iron was used if it was easier to manufacture a large shield. Sometimes concrete blocks were used to fill large areas near the bulk shield walls, to save cost. These SS have been proposed and used to lower radiation levels for other accelerator facilities [9] but were typically added for miss-steered beam losses from transport line dipoles. The LSDCG, however, analyzed the potential abnormal losses from all major magnetic elements in the transport lines and accelerators in the NSLS-II project and proposed SS's be installed wherever the radiation potential exceeded the NSP levels to occupied spaces.

The result of the LSDCG output was a document that specified the number, location, material, and design size and location specifications for the SS for each accelerator system. These Supplemental Shielding Design Documents (SSDD) were specified for the: Linac and LT1 transport line [10], the LTB transport, Booster and BS1 transport line [11], and the BTS transport and Storage Ring [12]. The specific SS design and simulated dose rate for the SSs were reviewed by the LSDCG and later by the Photon Science Directorate Radiation Safety Committee. The SSs were installed and tracked using a Quality Control Traveler document that verified the SSs met the requirements of the SSDD. Once installed properly, the verification of these critical devices function is managed by the PSD Configuration Management System and any work on the SSs are performed under a Safety System Work Permit, which assures that design configurations of the SSs are restored before return to service of the accelerator.

During the commissioning process of each accelerator, Radiation Fault Studies were used to verify the radiation levels outside the shield walls met the NSP and the simulated dose levels included in the SSDD. The comparison between the measured and simulated dose levels is presented in another paper [13], which showed agreement to better than $20 \%$, provided the beam loss location and charge lost was well known. This showed that the predictive power of the FLUKA program was valuable for insuring that the NSP would be met prior to commissioning and fault studies.

The methods used to identify these SSs, as well as some lessons learned in shielding the NSLS-II accelerators will be presented in this paper along with the shield designs and installation techniques used to adequately shield these three accelerators.

\section{Shielding Abnormal Beam Loss in NSLS-II Linac}

The NSLS-II Linac consists of four accelerating RF cavities with an operational accelerating voltage of $50 \mathrm{MV}$ for a klystron peak power of $42 \mathrm{MWatt}$ and $300 \mathrm{~ns}$ pulse length from each of two klystrons required to achieve the $200 \mathrm{MeV}$ operating energy. The nominal operating parameters for the Linac are $200 \mathrm{MeV}$ beam energy with a beam current of $15 \mathrm{nC} / \mathrm{sec}$. Higher energy is possible from the Linac up to $250 \mathrm{MeV}$, but is not planned for routine operations. If during nominal operations one of the two klystrons trips off, then the remaining klystron cannot provide the 200 $\mathrm{MeV}$ accelerating gradient and the beam output energy could drop to $100 \mathrm{MeV}$ or lower. However, it is also possible to shift the relative phases of the accelerating cavities and that the resulting beam energy can have any value between $200 \mathrm{MeV}$ and $0 \mathrm{MeV}$. The supplemental shielding was designed to handle full beam current losses at any energy between the $250 \mathrm{MeV}$ maximum and the $10 \mathrm{MeV}$ (injector energy) lower energy shield wall limit for $15 \mathrm{nC} / \mathrm{sec}$. Below $10 \mathrm{MeV}$, a beam current of 15 nA normally incident on the $1 \mathrm{~m}$ shield wall will generate an average dose rate of $<0.5 \mathrm{mrem} / \mathrm{h}(5$ $\mu \mathrm{Sv} / \mathrm{h}$ ) outside the shield, based on a FLUKA calculation. Therefore beam energies below $10 \mathrm{MeV}$ will be adequately shielded by the bulk shield walls and this energy will be considered an effective lower energy limit for the analysis of radiation levels for miss-steered beam. The Linac has two beam dumps; one in line with the Linac and the second for beam bent by the first dipole in the Linac to Booster Transport line (LBT). This dipole provides for energy analysis and energy selection of the Linac beam prior to transport to the booster. Both Linac dumps have their shielding designed 
using the ASM Eq. (2) for $\mathrm{E} \leq 230 \mathrm{MeV}$ and current $\leq 22 \mathrm{nA}$ [14]. The design of some of the Linac SSs [10] and some lessons learned are presented below.

1. Shielding Dipole Miss-steered Beam Losses

During commissioning the NSLS-II linear accelerator, beam energy was reduced to 100 $\mathrm{MeV}$ to study the beam properties when only one of two klystrons is available to accelerate the electron beam. While operating at this energy the first Linac to Booster Transport line dipole (LBTB1) was accidentally operated at full field bending the beam beyond the installed dipole supplemental shield (DSS), striking a concrete shield wall. Outside this shield wall occupancy is normally prevented (by PPS interlocks) when injecting to the booster accelerator. However at this time access was permitted for Linac commissioning and the radiation levels in the booster tunnel exceeded the values permitted by the NSP. The radiation levels outside the shield wall were studied and the DSS shield was extended to reduce these levels to acceptable levels. This incident clearly demonstrated that the entire range of operating conditions of the accelerators must be included when designing where and how big the DSS must be to prevent high dose levels to occupied area during abnormal conditions of the accelerators.

The DSS was originally designed to intercept miss-steered beam [14] for assumed beam energy of $140 \mathrm{MeV}$ (one klystron operation) and the dipole field at the operational value for 200 $\mathrm{MeV}$ beam energy. The LBT-B1 is a large aperture dipole with large non-uniform field away from the designed $12.89^{\circ}$ bend trajectory. The shielding limit was defined using a uniform field approximation of the bending which over estimates the bend angle compared to the tracked beam trajectory in the calculated non-uniform field of the magnet [15] as shown in Figure 6. These trajectories were tracked at $82 \%$ of the full field value and the beam energy of $80 \mathrm{MeV}$ just hit the downstream magnet yoke with a total bend angle of $46^{\circ}$. For the full field value this trajectory would have required $\mathrm{E}=97 \mathrm{MeV}$ to hit the yoke with the same bend angle. Figure 6 also shows that the originally installed SS would have also intercepted beam with energies E >158 MeV (at full field) not just the designed value of $\mathrm{E}>200 \mathrm{MeV}$. However if beam did hit the yoke, FLUKA simulations showed it was sufficient to reduce the radiation levels outside the shield wall to acceptable values [15]. Consequently the $\mathrm{E}=100 \mathrm{MeV}$ beam was bent $42^{\circ}$, which was just between these two shields and thus generated a radiation incident.

The result was to extend the DSS to the edge of the dipole yoke as shown in Figure 7. Only one direction of bend was covered even though the polarity wasn't guaranteed for this magnet, since the reverse bend direction had adequate shielding and restricted occupancy outside the shield wall (a 15 meter dirt berm and interlocked perimeter fencing). Another solution would have been to limit the power supply current to $70 \%$ of the maximum output with a PPS credited interlock but this wasn't chosen.

2. Shielding Quadrupole Miss-steered Beam Loss

Additional shields were added to allow the Linac to operate to the second of two beam dumps in the Linac vault while the booster tunnel is accessible for maintenance. Two credited protection devices were interlocked to prevent beam from being bent into the booster tunnel for this type of operation. One is a bending magnet LBT-B2 interlocked off, which must be energized to bend beam into the second half of the LBT and into the booster tunnel. The second device is a $30 \mathrm{~cm}$ Tungsten Safety Shutter must be inserted to block the transport line inside the Linac tunnel. However with Linac beam passing through the large aperture LBT-B2 dipole to the dump; it could be miss-steered by the LBT-Q6 quadrupole producing high radiation levels inside the booster tunnel. The QSSs necessary to shield this beam loss condition are shown in Figure 8. Another important shield was the Safety Shutter Mask, which prevents miss-steered beam from generating secondary electrons which could pass around the closed beam shutter and then be bent into the open beam pipe after the shutter where the LBT-Q9 quadrupole (not interlocked off like LBT-B2 dipole). This would allow a small area but high radiation level inside the booster tunnel through the open 
beam pipe. Whenever adding a safety shutter to block a beam pipe, a good radiation protection principle for high energy radiation fields is to insure the area around the beam pipe is similarly shielded as the shutter.

\section{Shielding Cable Tray Penetrations of the Shield Walls}

An important lesson was learned with regards to cable and waveguide penetrations between the occupied space and the Linac or Booster tunnels. The best practice is to insert cable conduit in the bulk shield walls with a bend to avoid line of sight for the radiation as was done in the NSLS-II storage ring. However for straight cable penetrations, the cables should have an elevation (bend) within the penetration so that shielding can be placed at both ends of the penetration to avoid line of sight for the radiation field. It is important that the elevation change of the cables in the penetration needs to be counter to the flow of the radiation, i.e. if the penetration is higher than the source of radiation then the cable needs to be high in the source tunnel and lower in the occupied area. Unfortunately this wasn't done in the Linac and Booster tunnels and this complicated the shielding of these penetrations and resulted in higher levels of radiation in the occupied areas. However since these were typically at an elevation of greater than $210 \mathrm{~cm}$, they weren't an issue for normal occupancy. However it could be an issue if maintenance was performed during beam operations for staff on ladders. Figure 9 shows one of the more complicated penetration shields developed in the Linac which caused some concern during fault studies [13]. The shield blocks added, Figure 9(b) required extending the shield below the cable tray into the Linac tunnel to minimize the entrance radiation into the penetration.

Figure 10 shows the FLUKA model for the LBT beam line in the Linac Tunnel with the magnetic elements and SSs as specified in the Linac SSDD [10]. Simulation of the expected dose levels for the most likely identified beam losses during operations and beam radiation fault studies have been analyzed for the range of energies from 10 to $250 \mathrm{MeV}$ and at a beam current of $15 \mathrm{nA}$. These were reported in the SSDD [10] and had maximum dose rates of less than $10 \mathrm{mrem} / \mathrm{h}(<100$ $\mu \mathrm{Sv} / \mathrm{h})$ to occupied areas.

\section{Shielding Abnormal Beam Loss in NSLS-II Booster}

The booster synchrotron is normally injected at $200 \mathrm{MeV}$ and accelerates beam to $3 \mathrm{GeV}$ with a $1 \mathrm{~Hz}$ repetition rate. The lattice consists of four arcs each with alternating gradient dipoles:7horizontal focusing dipole-BF $\left(3.3^{\circ}\right.$ bend angle $), 8$-horizontal defocusing dipole- $\mathrm{BD}\left(8.4^{\circ}\right.$ bend angle), 6-quadrupole and 4-sextupole magnets. The $158 \mathrm{~m}$ circumference ring is enclosed in a concrete tunnel with the injection arc of the tunnel surrounded by $15 \mathrm{~m}$ thick dirt berm and an interlocked interior courtyard shielded by $15 \mathrm{~m}$ thick dirt berm on the beam plane, see Figure 11. Arc2 has no outer berm but has an interlocked perimeter fence. The extraction straight is separated from the SR tunnel with an $80 \mathrm{~cm}$ concrete shield wall with the SR tunnel secured and interlocked during injection into the SR. However, access is permitted for maintenance during booster operations provided the Booster to Storage Ring Transport line (BST) is protected with two credited devices. The first is the BST-B2 dipole power supply locked out, preventing beam from being bent into the BSR beam line to the SR penetration and second is the BST Shutter is closed blocking this beam line penetration to the SR. Arc3 and 4 have an outer shield wall of $1 \mathrm{~m}$ concrete in order to shield the occupied Injector Service Area (ISA) which contains the equipment necessary to operate the booster and transport lines.

A PPS interlock was established on the Linac beam energy for injection, requiring that the LBT-B1 dipole to have a field value for transport of beam energy between 150 and $220 \mathrm{MeV}$. This limited the range of possible miss-steering angles that the LBT and the booster dipoles could generate. The maximum beam current in the LBT was limited less than $22 \mathrm{nA}$. The booster dipoles have a credited control that insures all dipoles have a field polarity that bends the beam inward, thus insuring the maximum outward angle is for zero field i.e. power supply off. The first dipole after 
injection is a BD dipole followed by a BF, therefore only these two magnets are relevant considering miss-steering the beam at zero field, since polarity checking also ensures all magnets in a string are powered to the same field. However there are two BD dipole power supplies with the BD magnets of Arc1 \& 2 on one and Arc3 \& 4 on the other power supply, placing similar beam loss requirements in Arc1 and 3 for the zero field injection loss. However since beam cannot be stored it also can't be accelerated, making these miss-steering losses only an issue for the injection energy range possible from the LBT transport line with its PPS interlock..

\section{Shielding Dipole Miss-steered Beam Losses in Booster Ring}

Within the parameters of the injection beam from the Linac and the maximum outward misssteering errors of the booster ring was determined by miss-steering of the injection or extraction bumps. The largest outward angle $\left(\sim 3^{\circ}\right.$ outward $)$ occurred after the first BD dipole in an Arc which required a larger DSS that collared the beam pipe after this BD dipole. The inner shield was required for injection septum off and $\mathrm{BD}$ dipole at maximum field ( $3 \mathrm{GeV}$ value). Although Arc1 and 2 would have had acceptable radiation levels at the fence limits without these DSS, it was decided to install them to avoid special configurations for these arcs. The subsequent dipoles had maximum outward steering less than or equal to the zero degree ray from Bremsstrahlung radiation which requires shielding for the $3 \mathrm{GeV}$ beam. Therefore DSSs were required to shield occupied areas for this radiation level from gas or beam striking an aperture at $3 \mathrm{GeV}$. For simplicity the first five DSSs were the same in all Arcs, as shown in Figure 12. Only Arc 2 and 3, which had the Bremsstrahlung radiation directed to the SR shield wall or the ISA shield wall, required additional 10-DSSs similar to the first 5-DSSs.

Booster extraction provide additional challenges for beam miss-steering since the pulsed nature of the extraction bumps and kickers has many possible combinations of angles and position of the 3 $\mathrm{GeV}$ beam hitting the chamber walls. With these possible beam loss locations directing EM showers toward the SR shield wall or the ISA shield walls, extra shields were required to both sides of the beam pipe downstream of the extraction septum. These are shown in Figure 13, which shows the forward and inner extraction shield in addition the outer and roof shields transverse to the extraction septum. With these shields installed the worst case simulated radiation levels inside SR tunnel was less than $22 \mathrm{mrem} / \mathrm{h}(220 \mu \mathrm{Sv} / \mathrm{h})$ and in the ISA less than $10 \mathrm{mrem} / \mathrm{h}(100 \mu \mathrm{Sv} / \mathrm{h})$ for $3 \mathrm{GeV} 15 \mathrm{nA}$ beam loss [11].

One final beam loss was determined to be a possible extraction bump magnet miss-firing to the $3 \mathrm{GeV}$ value after the 150 to $220 \mathrm{MeV}$ injection beam was circulating but not accelerated. This would kick the beam out of the extraction straight section and beyond the installed extraction shields as shown in Figure 14. This would generate radiation dose rates in the SR tunnel and near the ISA power cable penetration that would exceed the NSP level. For the low energy beams that would cause these radiation levels, a $2.54 \mathrm{~cm}$ steel plate to the outside of the bump region (ahead of the extraction septum) was sufficient to lower this dose rate to less than $20 \mathrm{mrem} / \mathrm{h}(200 \mu \mathrm{Sv} / \mathrm{h})$ in the SR tunnel.

\section{Labyrinth Design for Beam Losses in Booster Ring}

The need to minimize the labyrinth space and to provide access for passage of completed magnet girders for accelerator installation placed restrictions on the design of the large aperture labyrinth into the booster tunnel. The chosen design was a single dogleg labyrinth with an inner shield wall that was made of stackable high density concrete (HDC) which allowed initial access of the magnet girders and then blocks could be installed after the access was no longer required. Figure 15(a) shows the layout of the ISA to Booster labyrinth provided with the HDC blocks installed in the access way. Since the labyrinth has the entrance for radiation upstream of the exit, radiation from high energy beam loss that might hit the DSS in Arc4 will provide an intense 
radiation source near the booster tunnel side entrance into the labyrinth. The high energy components of this shower will enter the labyrinth in the booster tunnel and penetrate the corners of the labyrinth bends creating a high dose region on the downstream corner of the labyrinth exit into the ISA building. This is shown in Figure 15(b) for the dose distribution on the beam plane in the labyrinth for a $3 \mathrm{GeV}$ striking the DSS downstream of the $3^{\text {rd }} \mathrm{BD}$ dipole in the ring, where the high dose is seen at the ISA labyrinth exit doorway.

A better design would have been to interchange the location of the entrance and exits of the labyrinth in order for labyrinth flow to be counter to the HE radiation shower. This improved design is shown in Figure 16(a) for the labyrinth shielding layout and in Figure 16(b) for the dose distribution resulting from the same lose location as in Figure 15(b). This shows less penetration of the labyrinth inner bend corner since the shower has to back scatter from the shield wall. The dose distributions at the labyrinth exit into the ISA are shown in Figure 17 for both designs. Although the dose rate only decreases by a factor of 3-4, it is more uniform across the opening. This improvement would have been greater except that the thinner inner labyrinth wall was only 75 $\mathrm{cm}$, compared to the booster ISA shield wall thickness of 1meter. This allows more transverse radiation to penetrate directly to the exit opening. Special care is needed to insure that especially the inner walls of single dogleg labyrinth are at least as thick as the outer bulk shield walls which could be thinned in the overlap region of the labyrinth walls, but not on the inner walls.

Downstream of this labyrinth the forward shield wall was only $70 \mathrm{~cm}$ thick shield wall between the booster tunnel and the Klystron Gallery. This posed additional radiation issue for the more likely loss conditions at the injection energy. However this wall thickness wasn't identified until an injection beam fault study generated high levels for a $200 \mathrm{MeV}$ injection beam, which can be misssteered to larger angles. This condition was less of an issue for the $3 \mathrm{GeV}$ beam loss than it was for the labyrinth shielding study, since the DSS's intercepted more of the EM shower at $3 \mathrm{GeV}$ than for the injection beam energy. During the radiation fault study at $200 \mathrm{MeV}$ with the insertion of a vacuum gate valve into the beam chamber after the RF cavity, a small diameter high radiation level was observed after this shield wall. The detailed analysis with FLUKA (including magnetic fields for all dipole and quadrupole magnets using the magfld.f subroutine) showed that radiation levels at that wall would be high but the measured peak was a factor of ten times above the simulated values. The FLUKA dose levels around the peak were in agreement with the levels measured at those locations [13], making the peak dose value quite surprising and in agreement with a possible void in the shield wall. The solution was to increase the thickness of this shield wall with stacked concrete blocks, which lowered all dose rates at the wall by a factor of 8-10.

\section{Shielding Abnormal Beam Loss in NSLS-II Storage Ring}

The $3 \mathrm{GeV}$ beam from the booster ring is injected into the SR via the Booster to Storage Ring transport (BST) line. The SR lattice has 30 double bend achromatic cells with alternating long and short straight sections for insertion devices, giving a basic 15 -fold periodic symmetry to the lattice. The stored beam current ( $\left.\mathrm{I}_{\mathrm{av}} \leq 500 \mathrm{~mA}\right)$ is maintained by a $500 \mathrm{MHz}$ RF system which bunches the beam in trains of 1 to 1320 short bunches ( 12- 25 psec bunch length) of electrons separated by 2 nsec. The $792 \mathrm{~m}$ circumference SR is enclosed in a concrete tunnel with a nominal $1 \mathrm{~m}$ concrete outer wall (at $1 \mathrm{~m}$ from the ring vacuum chamber) and $80 \mathrm{~cm}$ roof and inner shield walls, each $2 \mathrm{~m}$ from the vacuum chamber. Parts of the inner wall have Service Buildings (SB) which provides HVAC and other services to the SR tunnel. The outer bulk shielding was designed using ASM Eq. (1) and for an assumed $3 \mathrm{GeV}$ operational beam loss rate of $1.68 \mathrm{nC} / \mathrm{min}$ in a thick target at the vacuum chamber $1 \mathrm{~m}$ from the wall [2]. The concrete thickness is $100 \mathrm{~cm}$ at the closest point to the vacuum chamber but as the vacuum chamber bends away from the outer wall, which is aligned with the X-ray beam line, it is thinned to $80 \mathrm{~cm}$. The forward ratchet walls, with the X-ray beam penetrations consist of $140 \mathrm{~cm}$ of concrete to shield for Bremsstrahlung radiation and miss-steered 
beam losses. The two cells around the injection region, the outer (experimental floor side) bulk shield walls have the same thickness as all other cells but they are made with high density concrete (HDC density 3.8 vs $2.35 \mathrm{~g} / \mathrm{cm}^{3}$ ), to allow for an operational beam loss rate of $0.28 \mathrm{nC} / \mathrm{s}$.

Beam is injected into the ring using a DC and pulsed septa, with a closed four pulsed kicker store beam bump. Once beam is injected and stored in the SR the total beam energy ( $\mathrm{J}$ ) is fixed. If the beam is lost the radiation exposure, although creating a high peak dose rate, will yield low average exposure due the longer time it takes to re-inject stored beam to that level. There are many ways that stored beam can be lost but the most likely losses will be from interlock trips and/or RF field tripped off and beam spiraling into the peak energy dispersion locations (i.e. between the two dipoles). This stored beam loss will generate high Bremsstrahlung radiation in the zero degree bend angle direction from the second dipole in the cell, which will be intercepted by the second DSS described below. With 30 identical locations of peak dispersion, the radiation exposure at any one location will typically be reduced by 30 fold loss locations. The one difference to this 30 fold distributed loss is the use of the scrapers to limit the loss to the injection period, which will be discussed below. For stored beam losses other than RF system trips, the losses will similarly be distributed since magnet field steering errors will develop slowly compared to the revolution period and will create Betatron oscillation around the SR with more than 30 peak positions around the SR. These will strike the vacuum chamber at several locations similarly distributing the beam energy losses. The one exception to this is the corrector magnets which are faster but quite weak (steering the beam by a maximum of 0.9 mradian) therefore requiring multiple correctors to miss-steer the beam to one location which reduces the likelihood of such miss-steering. Therefore the stored beam loss represents a smaller radiation risk than that from injection beam miss-steering which although lower in energy per pulse, could present a continuous rate of beam loss that could continue indefinitely, unless terminated by an Area Radiation Monitor (ARM) interlock level or by operator intervention. This represented the greatest radiation risk and was the beam loss scenario of most concern to the LSDCG. However, shielding the injection beam loss locations also provides shielding for the most likely stored beam loss locations as well.

To analyze the possible loss location for injected beam, the LSDCG specified a PPS interlocked energy selection on the injection energy to be set in the BST beam energy limit to $3 \mathrm{GeV} \pm 5 \%$ using the first two dipole magnets (BST-B1 \& B2) in the transport line. This insured that injection beam had $2.85<\mathrm{E}<3.15 \mathrm{GeV}$ for efficient transport to the SR [16]. Similarly the SR dipole field was also included in the PPS system to limit the acceptable energy to $3 \mathrm{GeV} \pm 2 \%$. As previously stated a credited control of the bending polarity of the SR dipoles was implemented. The SR dipoles are $\mathrm{C}$-shaped magnets with the return yoke toward the inside of the ring. Without an iron yoke to the outside of the ring, shielding might be considered necessary outside every dipole to protect against an accidental dipole polarity reversal. This credited control was crucial for protection the SR experimental floor from a reversed polarity dipole. To determine the possible miss-steering angles, all other magnets were scanned for both polarities since this is possible for injection but not for stored beam. All magnets had the maximum angles for miss-steered beam loss determined but the quadrupole angles were smaller and typically were intercepted by the yoke of subsequent magnets, than for the dipoles. Both dipoles had similarly large angles for miss-steered trajectories that even exceeded outward angle of the input reference angle (i.e. zero bend angle) as is shown in Figure 5. There are even trajectories with the zero degree bend angle (X-ray beam direction from ID), however they will be intercepted by photon beam apertures, photon absorbers or fixed masks which provide an EM shower source location but not sufficient shielding to attenuate or spread the beam energy. These dipole miss-steering locations were the greatest concern of the LSDCG and resulted in specifying of 60 dipole DSS discussed below.

1. Shielding of Dipole Miss-steered Beam Losses in Storage Ring 
Initially there was a specification for DSSs downstream of every SR dipole to intercept the Bremsstrahlung radiation from the beam. These were specified to be $15 \mathrm{~cm}$ thick Pb DSS [17]. A Radiation Safety Review proposed a revision to the DSS design suggesting that they could be incorporated into the forward ratchet wall bulk shield $[18,19]$. They were expanded to $25 \mathrm{~cm}$ thickness of $\mathrm{Pb}$ covering the entire horizontal front surface ( 1.5 to $2.1 \mathrm{~m}$ long) of the ratchet wall and $50 \mathrm{~cm}$ vertically, using ASM Eq.(2). When the geometry of the shield wall was included in the FLUKA simulations, the EM shower from miss-steered injection beam hitting a thin target (a photon absorber or vacuum flange in an unused beam port or a closed dipole photon shutter in an installed beamline) the EM shower exceeded the height of the lead shield in the ratchet walls. Consequently the dose rate downstream of the ratchet wall had extremely high dose rates ( $30 \mathrm{rem} / \mathrm{h}$ or $\sim 0.3 \mathrm{~Sv} / \mathrm{h}$ for a $15 \mathrm{nA}$ injection beam loss) for vertical distances $>30 \mathrm{~cm}$ above and below the beam plane for the unused beam-port ratchet walls as shown in Figure 18 and above the beam plane for installed beam lines (the girder supports reduce the lower peak). Therefore the LSDCG recommended installing 30cm thick Pb DSS's downstream of each SR dipole with at least 45 mradians (plus 3 Moliere lengths $3.8 \mathrm{~cm}$ ) outward angular coverage relative to the X-ray photon beam direction to ensure intercepting all miss-steered trajectories out of the dipoles. The bulk shield wall after the first dipole restricted the outer angle of the DSS as shown in Figure 19, but with a clever support design, a location was found after the unused beam line flange that allowed > 90 mradian coverage to the limit of the shield wall angular coverage. The second dipole had no such restriction but its DSS had to be placed further from the dipole but was within $1.8 \mathrm{~m}$ of the unused beam line flange. The layout for both DSS's after the dipoles are shown in Figure 20 with the X-ray beam pipe installed and passing through the DSS. Figure 21 shows the dose rate outside the ratchet wall for the unused beam line with the beam hitting the absorber and flange as shown in Figure 18 but with the $30 \mathrm{~cm}$ DSS installed $80 \mathrm{~cm}$ after the flange. The peak dose above and below the mid plane has been almost completely eliminated with a reduction of $\sim 1000$ times for the total dose and $~ 500$ times for the neutron component. A small increase in dose is seen near the floor in Figure 21 (a), since the DSS was not centered on the beam plane but shifted high by $5 \mathrm{~cm}$ in order to better shield the mezzanine above the ratchet wall. This DSS shielding demonstrates a basic principal of shielding, that of locating the shield as close as possible to the loss point in order to increase the angular cone of the EM shower, soften the particle energies and absorb energy within the shower before hitting the shield walls. This also contributes to lower shielding costs by reducing the transverse size of the shield. Additionally the SS is more effective per unit of thickness when the shield is nearer to the target (far from the wall, as shown below.

This principle is demonstrated more simply by a FLUKA simulation of a $3 \mathrm{GeV}$ electron beam hitting a thin $1.8 \mathrm{~cm}$ thick Copper target (similar to dipole photon shutter) and the forward ambient dose equivalent calculated a distance $\mathrm{R}=10 \mathrm{~m}$ downstream after a $100 \mathrm{~cm}$ concrete shield wall. A $30 \mathrm{~cm}$ thick cylindrical $\mathrm{Pb} S S$ is then placed either near to $(\mathrm{z}=50 \mathrm{~cm}$ with radius $\mathrm{r}=15 \mathrm{~cm})$ or far from the target $(\mathrm{z}=870 \mathrm{~cm}$ with radius $\mathrm{r}=100 \mathrm{~cm})$. To compare with Tables I and II and the ASM Eq.(2) the ambient dose equivalent is normalize by $\mathrm{R}^{2}\left[\mathrm{~m}^{2}\right]$ and divided by the total beam energy $\mathrm{J}$ [Joules]. The geometry for the near SS is shown in Figure 22 along with the internal dose distribution. These shielded ambient dose equivalent values are listed in Table III for near and far SS locations. The dose levels for the SS near to the target are 15-30 times lower than for the SS far from the target. This is the result of the small EM shower angular divergence from the target and the resulting secondary source term in the SS which is isotropic for neutrons. This secondary source term in the SS has large solid angle differences at the dose scoring location between when the SS is near to and far from the target. A secondary source term in shields is not included in the ASM Eq. (2) and although not a significant effect for transverse dose from a thick target, as shown in Table II, the forward thin target beam loss shows a major difference sufficient to greatly exceed the conservative nature of the ASM estimates, as shown in Table III. 
2. Shielding Beam Losses in the Injection Period of the SR

The most likely abnormal miss-steering of the injection beam and the greatest risk, will occur in the injection region, where the pulsed septum and kickers could miss-fire. These magnets must not only pulse to the correct value but also pulse at the correct time to properly steer the beam into the SR vacuum chamber. In addition the small aperture of the septum may contribute to significant miss-steering losses even when the pulsed magnets are working properly. The ASM Eq. (2) estimated the thickness of lead shielding that was needed for a $20 \%$ operational beam loss to be 10 $\mathrm{cm}$ in all transverse directions [19]. However for $100 \%$ miss-steered beam loss the FLUKA dose estimates required the outer septum shield to increase to $25 \mathrm{~cm}$ of lead due to the more forward directed trajectories when the pulsed and DC septa are off. The inner wall and roof shields were not increased in thickness. However, the roof thickness was changed to a $20 \mathrm{~cm}$ thick steel plate instead of lead, since steel is self-supporting and easier to install as a ceiling shield. The original concept was to make two right angle shields (half top and one side) that were supported by overhead girders on trollies to allow them to be retracted for maintenance of the pulsed magnets and their power supplies under magnets. However with the change to a steel roof shield the entire transverse span over the injection region could be made one piece, eliminating the seam above the middle of the beam path. The two side curtains of lead bricks were supported in a steel frames with aluminum covers. Figure 23 shows the FLUKA model of the SR injection region shield. Figure 24 shows the advantage of this three component design (roof and two side curtains each on separate trollies) with all three components retracted for access to the pulsed magnets. However for access to only one side of the magnets, only one curtain needs to be retracted.

Even with the injection septum shields in place there are still high radiation levels in the bulk shielding near the sliding access door. With the bulk shield walls in the injection region made of HDC, they have 50\% more attenuation than the normal cell shield walls. These composite doors were calculated to be the equivalent to the $80 \mathrm{~cm}$ normal concrete using the ASM Eq. (2) [17] and therefore are not as effective as the HDC walls in the injection region. Figure 25(a) shows the dose outside the cell 29ID door for the beam loss condition where the DC septum has tripped off. The dose at the door body is 3-5 times the dose at the wall ahead of it, with the downstream crack of the door even higher. From the ALARA point of view it was decided to reduce the levels outside these doors. This was done by filling the inner doorway opening with hand stacked concrete block, which lowered this simulated dose rate a factor of 8-12 at the door and the crack, as shown in Figure 25(b).

The NSLS-II anticipated the lower lifetime and therefore high beam loss rate at high stored currents, by adding a beam Loss Control and Monitoring system (LCM) that was designed to control the beam losses (for beam dumps as well as lifetime losses) to the more heavily shielded injection region and to monitor (verify) the loss in that region [20]. This was done using variable position thin beam scrapers in the first two cells after injection to control the beam loss location during high current operations. In order to verify that the loss was in this region the scraper had to be thin enough to cause the beam to lose sufficient energy in the scraper so that the next dipole would bend the lower energy electrons out of the vacuum chamber, where Cerenkov beam loss monitors (CBLM) measured the number of electrons in the beam lost.

A series of five beam scrapers three horizontal and two vertical were installed in cell 30 (first after injection), shown in Figure 26, and also in cell 1. Only two of the horizontal scrapers located near the dispersion peak in these cells will be used for high current beam loss control. The others will be used for physics measurements, typically at lower currents. The scrapers are only $10 \mathrm{~mm}$ copper blades ( $70 \%$ of radiation length), creating a relatively low level radiation point source which doesn't require local shielding. However the thin scrapers absorb little of the beam energy and the subsequent dipole bends the residual electron beam into their inner yoke, where the CBLM 
are inserted. The level of radiation back scattered from this distributed source location inside the dipole will be shielded by the dipole yoke, but that which passes through the gap needs to be shielded. The positrons generated in the scraper will be bent to the outside of the ring by the dipole and will also require shielding transverse to the dipole, in addition to the already discussed DSSs which will intercept the Bremsstrahlung radiation and large scatter electrons from the scraper. Figure 27 shows the FLUKA model of the LSDCG specified scraper shields for the second dipole in Cell 30 which follows the horizontal scraper 1. This scraper is of greatest concern since the visible synchrotron light monitor takes light from that dipole to the Cell 30ID hutch, using $90^{\circ}$ mirrors. Since the DSS for this dipole has a beam pipe in it followed by a mirror, a SS was added after the mirror to absorb the forward radiation from the mirror.

The dipoles following the scrapers required $10 \mathrm{~cm} \mathrm{~Pb}$ side shields to attenuate the radiation from the dipole when the scraper is intercepting beam. Due to the wide vacuum chamber in the dipoles this lead couldn't be installed closer to the dipole gap than $\sim 38 \mathrm{~cm}$. This made the height taller than otherwise it could have been but a $20 \mathrm{~cm}$ high standard $\mathrm{Pb}$ brick was more than adequate at this transverse spacing. A shield length of $150 \mathrm{~cm}$ was found to adequate to cover the downstream portion of the $264 \mathrm{~cm}$ long dipole gap, since the upstream radiation field had lower energy components from this distributed beam loss source.

The simulated dose rates outside the shield walls for $15 \mathrm{nA}$ beam hitting horizontal scraper 1 using the FLUKA model shown in Figure 27. The dipole had a magnetic field to bend the $3 \mathrm{GeV}$ electron beam the required $6^{\circ}$. No additional magnets were modeled and the quadrupole and sextupole ahead of the dipole will cause some additional deflection of the low energy charged particles but these should be a small fraction of the dose for these thin scrapers. Figure 28 shows the particle fluence from the scraper generated components (electrons, positrons, gamma rays and neutrons) for the beam loss condition. The electron, positron and neutron components are well captured by these shields for the outward radiation fields which of greatest concern due to the user occupancy factor. The gamma component, Figure 28(c), has some fluence missing these shields (especially since the DSS is so far downstream due to quadrupole magnet interference) however these are lower energy gamma rays and easily attenuated by the bulk shield. The neutron fluence, Figure 28(d), plot clearly shows the lower production rate from the thin scraper and the higher secondary source from the dipole yoke, the DSS and the mirror SS. Figure 29 shows the fluence in a vertical plane through the dipole downstream end for gamma rays and neutron. The dipole side shield is quite adequate for the gamma rays (also electrons and positrons) but the neutrons show a much broader distribution with less attenuation in the $\mathrm{Pb}$ shield. As with the beam loss in the injection straight section and the dose outside the 29ID door, the 30ID door had similarly high dose rates for the scraper beam loss condition and this doorway was also filled with concrete blocks to insure ALARA conditions were met.

\section{Shielding Access Doors to the Experimental Floor}

As discussed above, the sliding access door to the experimental floor have had high levels penetrating the downstream ends. This was the result of thinning the door jamb on that end to only $50 \mathrm{~cm}$ of concrete. The overlap of the door and the jamb was $30 \mathrm{~cm}$ but this wasn't sufficient for the high energy showers from beam losses along the beam line or hitting the second dipole DSS to prevent high levels on the experimental floor. There were essentially three short path lengths through the present door jamb with less than $60 \mathrm{~cm}$ of concrete attenuation, as shown in Figure 30.The high levels outside these doors was measured during SR Radiation Faults Studies and a solution proposed [21,13]. This radiation issue is a direct effect of the use of the ASM and standard formulas for shield overlap for transverse thick target radiation fields, which fails to adequately account for the geometry of the high energy forward radiation in a shower. A better solution to providing these access doorways near the beam line front ends is shown in Figure 31 
which slides the door the opposite direction and increasing the downstream door jamb thickness plus an added pocket for the door to slide against. Figure 32 compares the radiation levels outside the door for the beam loss hitting the $2^{\text {nd }}$ DSS in a cell [21]. This new door design lowered the highest dose rates by factors of 80 to 100, even lower than the dose rates at the shield wall upstream of the door.

\section{Summary and Conclusions}

The LSDCG has spent considerable effort to fully analyze the potential abnormal beam loss locations and the radiation risks they impose of all three NSLS-II accelerators. Although the ASM was useful in specifying the bulk shield wall thickness it was based on an optimistic operational beam transport efficiency and assumed loss to a thick target at the vacuum chamber. These were demonstrated to be conservative estimates of the dose rate (over estimates the dose compared to FLUKA estimated dose rate) for these loss conditions. However abnormal beam losses don't necessarily meet these conditions and often can pass through thin vacuum components which will not generate a thick target shower. The high energy forward radiation shower from these thin targets needs to be attenuated and spread by SSs before it strikes the shield walls or the ASM conservative factor may become a significant underestimate of the actual dose. The geometric specification between the thick or thin target and transverse or forward directions are not easily dealt with in the ASM. The critical nature of this radiation problem is more than meeting a projected target with big uncertainty but of reducing the real exposure to ALARA levels. This requires better estimates of the real beam loss locations to be shielded and accurate estimates of the dose rate for the geometry of the real accelerator and installed shielding.

For State-of-the-Art low emittance SLS designed with State-of-the-Art accelerator codes with low beam lifetime and Top-Off operations, their radiation protection will require accurate estimates of the dose levels for most likely beam losses which require State-of-the-Art radiation shielding calculations. The FLUKA-FLAIR programs provide such a basis for estimating and minimizing the expected radiation levels to the users and staff both for normal and abnormal operations. The supplemental shield designed by the NSLS-II LSDCG provided such a minimization of the estimated dose outside the bulk shield walls, which has been verified by radiation fault study measurements in many cases [13].

Some of the lessons learned by the LSDCG and NSLS-II project that could benefit the shielding of future light sources are:

1) The ASM is useful to guide shield wall specifications for thick target beam losses, but is not sufficient to deal with thin target beam loss which could exceed its estimated dose level.

2) For thin target beam losses the geometry of the material objects in the beam loss path needs to be adequately defined in order to understand the width of the EM shower and the sizes needed for SSs to meet NSP levels.

3) Include the full range of operating energies and currents possible from each accelerators and the magnet power supplies in the analysis of the miss-steering of the beam losses,

4) Always install the SSs as close as possible to the loss location from the vacuum chamber in order to enhance the effectiveness of the shields and reduce cost of shielding.

5) Always install cables bends in straight penetrations and labyrinths with a single dogleg counter to the flow of the radiation from the sources.

6) Recommendations from reviews should be taken as suggestions but their real value needs to be analyzed and understood by the project, which has the responsibility for the safety of the facility. 


\section{Acknowledgements}

This paper summarizes the work of the LSDCG members: R. Fliller, P.K. Job, Y. Li, S. Seletskiy, and W. Guo, in addition to the authors. We also acknowledge the assistance of other NSLS-II staff: T. Shaftan, W. Casey, E. Johnson, R. Lee and D. Beavis. This large effort could not have been achieved without the continuing support and encouragement of F. Willeke, S. Sharma and S. Dierker. All this design and planning effort would have achieved little without the dedicated and competent efforts of the NSLS-II Design Group under M. Loftus and the Mechanical support Groups under R. Scheuerer and T. Dilgen, who turned this shielding effort into a reality. We also acknowledge efforts of the FLUKA-FLAIR Team for providing a modern tool for the estimating the radiation levels of real accelerators with relative ease and a high level of predictability.

\section{Glossary of Acronyms}

ALARA- As Low As Reasonably Achievable ASM- Analytic Shielding Model BST- Booster to Storage Ring Transport line CBLM- Cerenkov Beam Loss Monitors DSS- Dipole Supplemental Shield EM- electro-magnetic HDC- High Density Concrete ID- Insertion Device ICRU- International Commission on Radiation Units \& Measurements LBT- Linac to Booster Transport line LCM- Loss Control and Monitoring system LSDCG- Local Shielding Design Coordinating Group NSP- NSLS-II Shielding Policy PPS- Personnel Protection System

SLS- Synchrotron Light Source SS- Supplemental Shield

SSDD-Supplemental Shielding Design Document 
Table I The values used for the Dose Equivalent Factors, Fi used for NSLS-II shield wall thickness specifications and the FLUKA calculated unshielded scaled dose rates from a $30 \mathrm{~cm} 10 \mathrm{ng}, 10 \mathrm{~cm}$ diameter iron target. The units for Dose Equivalent Factors are $\mu \mathrm{rem} \cdot \mathrm{m}^{2} / \mathrm{J}$ or $0.01 \mu \mathrm{Sv} \cdot \mathrm{m}^{2} / \mathrm{J}$. The parameters for a power of $\mathrm{E}$ are from a fit to calculated doses versus beam energy.

\begin{tabular}{|c|c|c|c|c|c|c|}
\hline \multicolumn{7}{|c|}{ Unshielded dose at $\mathrm{R}=1 \mathrm{~m}$ from target } \\
\hline \multirow[b]{2}{*}{$\begin{array}{l}\text { Radiation } \\
\text { Component }\end{array}$} & \multicolumn{3}{|c|}{ Transverse $90^{\circ}\left[\mu \mathrm{rem} * \mathrm{~m}^{2} / \mathrm{J}\right]$} & \multicolumn{2}{|c|}{ Forward $0^{\circ}\left[\mu \mathrm{rem} * \mathrm{~m}^{2} / \mathrm{J}\right]$} & \multirow[b]{2}{*}{ power $\mathrm{E}$} \\
\hline & $\begin{array}{l}\text { Anal. } \\
\text { Dose } \\
\text { Factors, } \\
F_{i}[2,3]\end{array}$ & $\begin{array}{l}\text { FLUKA } \\
\text { Amb74 } \\
F_{i}(3 G e V)\end{array}$ & power $\mathrm{E}$ & $\begin{array}{l}\text { Anal. Dose } \\
\text { Factors, Fi } \\
{[4]}\end{array}$ & $\begin{array}{l}\text { FLUKA } \\
\text { Amb74 } \\
{\left[\begin{array}{ll}x & 10^{3}\end{array}\right]}\end{array}$ & \\
\hline Total Dose & 1693 & $1149 \pm 15$ & $0.02 \pm 0.015$ & $8.33 \times 10^{6} \mathrm{E}$ & $5.922 \mathrm{E}$ & $0.81 \pm 0.02$ \\
\hline Gamma Dose & 1380 & $535.5 \pm 2.6$ & $-0.026 \pm 0.02$ & $8.33 \times 10^{6} \mathrm{E}$ & $3.469 \mathrm{E}$ & $0.79+0.02$ \\
\hline Neutron Dose & 313 & $436.5 \pm 9.7$ & $0.29 \pm 0.03$ & 405.5 & 0.102 & $0.43 \pm 0.04$ \\
\hline \multirow[t]{2}{*}{ Charge Part. } & & $176.6 \pm 18$ & $-0.22+0.04$ & & $2.419 \mathrm{E}$ & $0.84 \pm 0.03$ \\
\hline & & & & \multicolumn{2}{|c|}{$\mathrm{E}$ in $\mathrm{GeV}$} & \\
\hline
\end{tabular}

Table II The normalized ambient dose equivalent levels (Amb74), at a distance $\mathrm{R}=2 \mathrm{~m}$ from the target, penetrating a $\mathrm{t}=1 \mathrm{~m}$ thick concrete shield wall transverse to the target. The doses were scored for the shield wall near to or far from the target

\begin{tabular}{|l|c|l|l|l|l|}
\hline \multicolumn{5}{|c|}{ Dose Equiv at R= $2 \mathrm{~m}$ Shielded with $1 \mathrm{~m}$ concrete $\left(\rho=2.35 \mathrm{~g} / \mathrm{cm}^{3}\right)$} \\
\hline \multirow{2}{*}{$\begin{array}{l}\text { Radiation } \\
\text { Component }\end{array}$} & $\begin{array}{l}\text { Transverse } 90^{\circ} \\
\text { Analytic } \\
\text { Dose } \\
\text { Equiv* }\end{array}$ & $\begin{array}{l}\left.\text { Dose Equiv } \mathrm{m}^{2} / \mathrm{J} \text { or } 0.01 \mu \mathrm{Sv} * \mathrm{~m}^{2} / \mathrm{J}\right] \\
\text { near } 3 \mathrm{GeV}\end{array}$ & $\begin{array}{l}\text { power } \\
\text { Energy }\end{array}$ & $\begin{array}{l}\text { Dose Equiv } \\
\text { far } 3 \mathrm{GeV}\end{array}$ & $\begin{array}{l}\text { power } \\
\text { Energy }\end{array}$ \\
\hline \hline Total Dose & 13.32 & $2.80 \pm 0.83$ & $0.60 \pm 0.07$ & $2.41 \pm 0.33$ & $0.44 \pm 0.05$ \\
\hline Gamma Dose & 11.40 & $0.42 \pm 0.11$ & $0.025 \pm 0.03$ & $0.52 \pm 0.07$ & $0.031 \pm 0.04$ \\
\hline Neutron Dose & 1.92 & $1.98 \pm 0.54$ & $1.12 \pm 0.05$ & $1.60 \pm 0.18$ & $0.76 \pm 0.06$ \\
\hline Charge Part. & & $0.395 \pm 0.35$ & & $0.29 \pm 0.3$ & \\
\hline \multicolumn{7}{|c|}{} & & & & \\
\hline * ASM Attenuation lengths for concrete used: gamma $\lambda_{\gamma}=20.85 \mathrm{~cm}$, \\
low energy neutron $\lambda_{\text {len }}=17 \mathrm{~cm}$, and high energy neutron $\lambda_{\text {hen }}=27.7$ \\
\hline
\end{tabular}


Table III The FLUKA simulated shielded normalized ambient dose equivalent levels at 3 $\mathrm{GeV}$ in the forward direction a distance $\mathrm{R}=10 \mathrm{~m}$ from a thin target $(\mathrm{t}=1.8 \mathrm{~cm} \mathrm{Cu})$. The target is followed by a cylindrical $\mathrm{Pb} \mathrm{SS}$ (radius $\mathrm{r}=15$ or $100 \mathrm{~cm}$ ) of thickness $30 \mathrm{~cm}$ and then a $1 \mathrm{~m}$ thick concrete shield wall. The data are for the SS placed either near to $(\mathrm{z}=50 \mathrm{~cm})$ or far $(\mathrm{z}=870 \mathrm{~cm})$ from the center of the target.

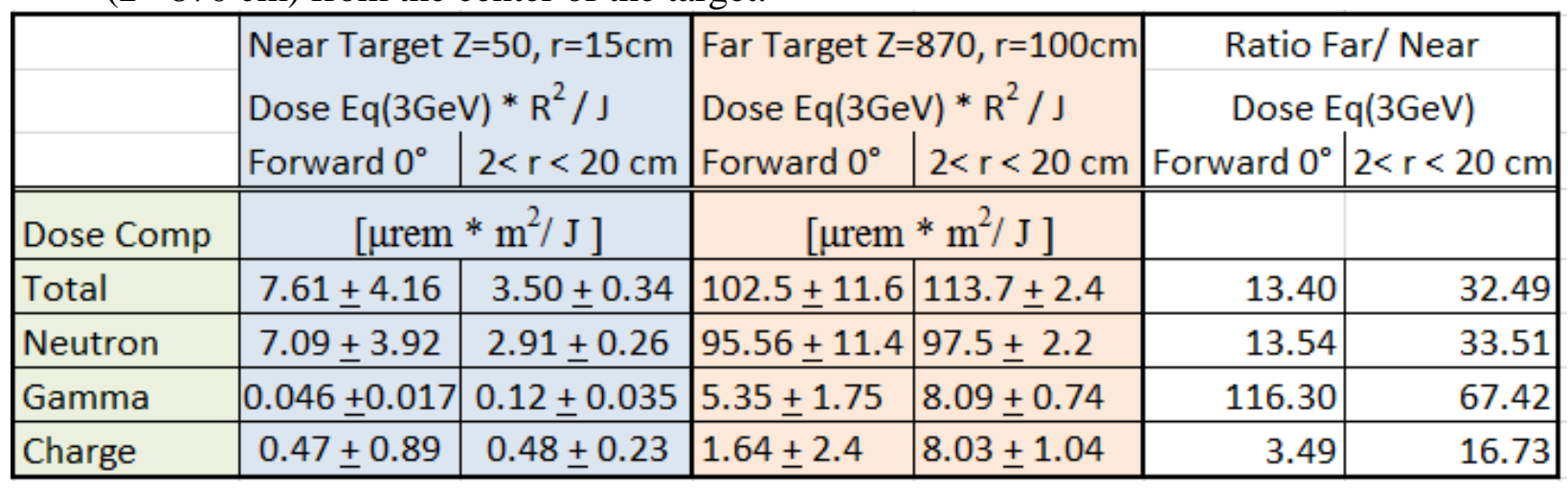

\section{Reference:}

[1] S. Ozaki, et. al., PAC 2007, BNL-79159-2007-CP, (2007) and S. L. Kramer, et.al. APAC 2007 (2007).

[2] Panakkal K. Job and William R. Casey, Nucl. Instr. and Meth. A660, p. 1 (2011).

[3] W.P. Swanson, "Radiation Safety Aspects of the Operation of Linear Accelerators", IAEA Tech. Report 188 (1979),

A.H. Sullivan, "The Shielding of an Electron-Positron Injector", CERM-LEP-354 (1982).

[4] A.H. Sullivan, "A guide to Radiation near High Energy Particle Accelerators", Nuclear

Technology Publishing (1992).

[5] FLUKA: a multi-particle transport code developed at CERN by Alfredo Ferrari, Paola R. Sala, Alberto Fasso and Johannes Ranft, https://www.fluka.org,

A. Ferrari, P.R. Sala, A. Fasso`, and J. Ranft, "FLUKA: a multi-particle transport code" CERN2005-10 (2005), INFN/TC_05/11, SLAC-R-773.

[6] M. Pelliccioni, Radiation Protection Dosimetry 88, p. 279 (2000).

[7] S.L. Kramer et. al., "Local Radiation Shielding Design Methodology", NSLS-II Tech Note 101 (2013), https://ps.bnl.gov/sites/tn/default.aspx and

Y. Li and S. Seletskiy, "Analysis of mis-Steered Electron Beam in the NSLS-II Storage Ring", Tech Note 122 (2013), https://ps.bnl.gov/sites/tn/default.aspx .

[8] NSLS-II Shielding Policy, PSD-C-ASD-POL-005 (2014).

This policy requires one radiation protection device (e.g. area radiation monitor-ARM) for dose rates $>100 \mathrm{mrem} / \mathrm{hr}$ and second credit control for rates $>2000 \mathrm{mrem} / \mathrm{hr}$.

[9] Y. Asano, "Shielding designs for the electron transport line of XFEL facility, SACLA to SPring8 storage ring", Radsynch 2013, https://www.bnl.gov/radsynch13/papers.php, (2013).

[10] Linac SSDD, LT-C-ASD-RSI-LIN-001 (2103), NSLS-II Accelerator Division safety documents, https://ps.bnl.gov/acc/Supplemental_Shielding/Shared\%20Documents/Forms/AllItems.aspx .

[11] Booster SSDD, LT-C-ASD-RSI-BST-001 (2013), NSLS-II Accelerator Division safety documents, https://ps.bnl.gov/acc/Supplemental_Shielding/Shared\%20Documents/Forms/AllItems.aspx . [12] Storage Ring SSDD, LT-C-ASD-RSI-SR-001 (2013), NSLS-II Accelerator Division safety documents, https://ps.bnl.gov/acc/Supplemental_Shielding/Shared\%20Documents/Forms/AllItems.aspx . [13] S.L. Kramer et. al., "Shielding Synchrotron Light Sources: Prediction and Verification of Radiation Levels from Electron Beam Losses", BNL-111851-2016-JA (2016). 
[14] P.K. Job, "Supplementary Shielding Specifications for the NSLS-II Linac Enclosure -Rev 2", NSLS-II Tech Note No. 88 (2012).

[15] S.L. Kramer et. al., "Supplemental Shielding for the LBT-B1 Dipole”, Tech Note 94 (2102) and Tech Note 127 (2013).

[16] S. Seletskiy, BNL-101365-2013-IR and BNL-101037-2013-IR, (2013).

[17] P.K. Job and W. Casey, "Shadow Shields in the Storage Ring of NSLS II", NSLS-II Tech Note 21, (2006) and Tech Note 13 (2007).

[18] "Review of NSLS2 Preliminary Shielding Calculations", 2007 Radiation Safety Workshop Final Report" (2007).

[19] P.K. Job and W. Casey, "Preliminary Material Requirement for the Supplementary Shielding at NSLS-II” Tech Note 32 (2007).

[20] S.L. Kramer "Loss Control and Monitoring System for NSLS-II", Tech Note 181 and "Beam Loss Monitoring for NSLS-II", proceedings of PAC2011, (2011).

[21] S.L. Kramer and M. Breitfeller, " SR ID Doorway High Radiation Levels and Proposed Shielding”, Tech Note 170 (2015) and

S.L. Kramer et. al., "SR Phase 3 Radiation Fault Study and FLUKA Estimates", NSLS-II Tech Note 155 (2015). 

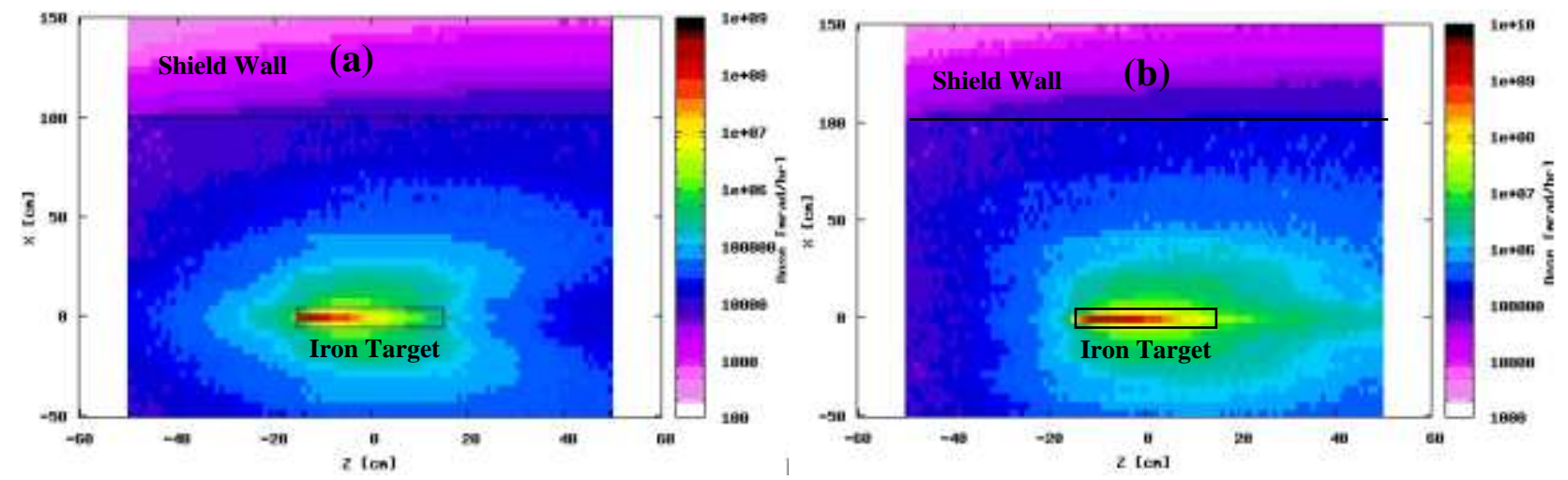

Figure 1 The FLUKA estimated dose distributions on the beam plane for (a) $200 \mathrm{MeV}$ and (b) 3 $\mathrm{GeV}$ electrons hitting the iron target.
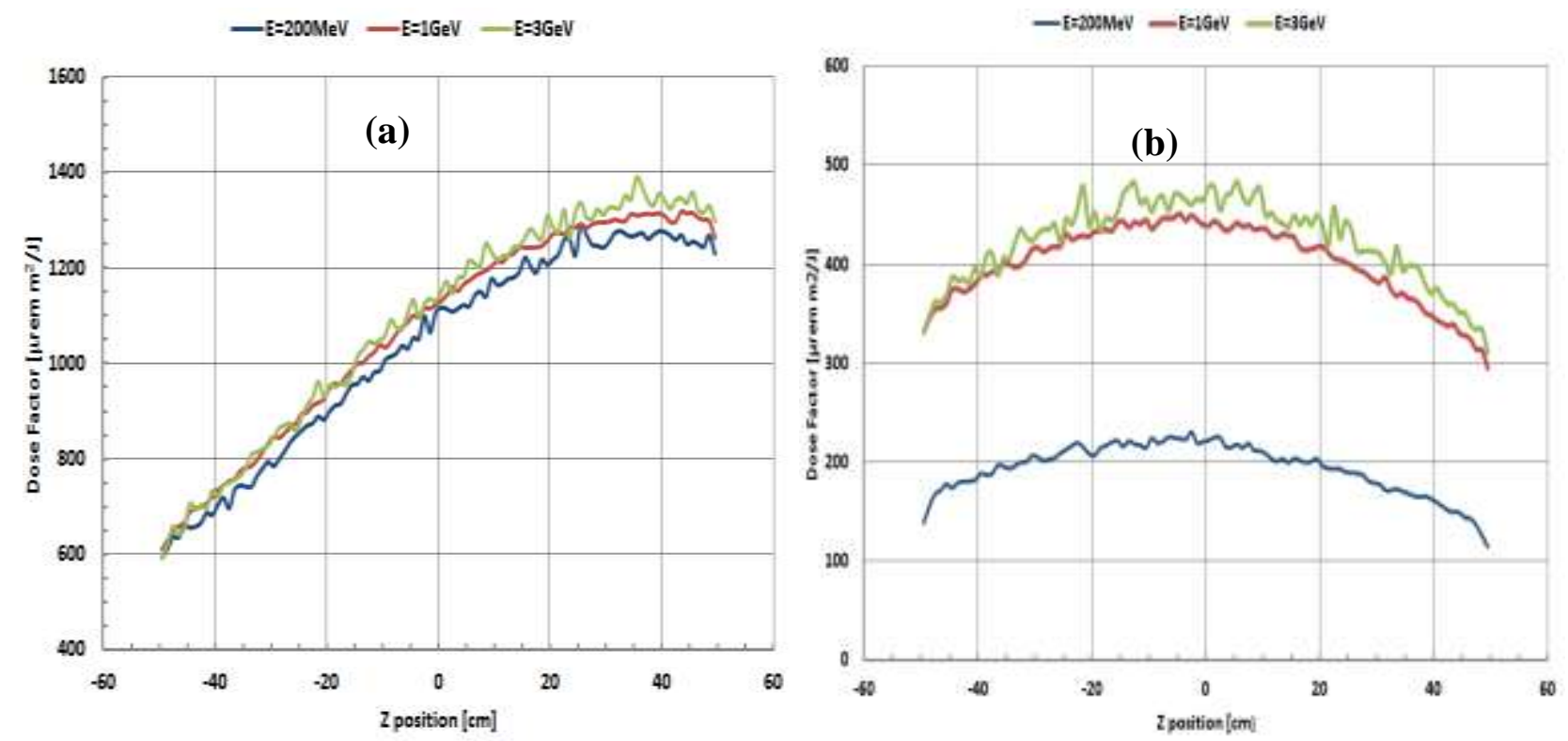

Figure 2 The transverse normalized ambient dose equivalent, ( $\mu$ rem $* \mathrm{~m}^{2} / \mathrm{J}$ or $* 0.01 \mu \mathrm{Sv}^{*} \mathrm{~m}^{2} / \mathrm{J}$ ), for (a) total and (b) neutron components for different energies, calculated by FLUKA. 

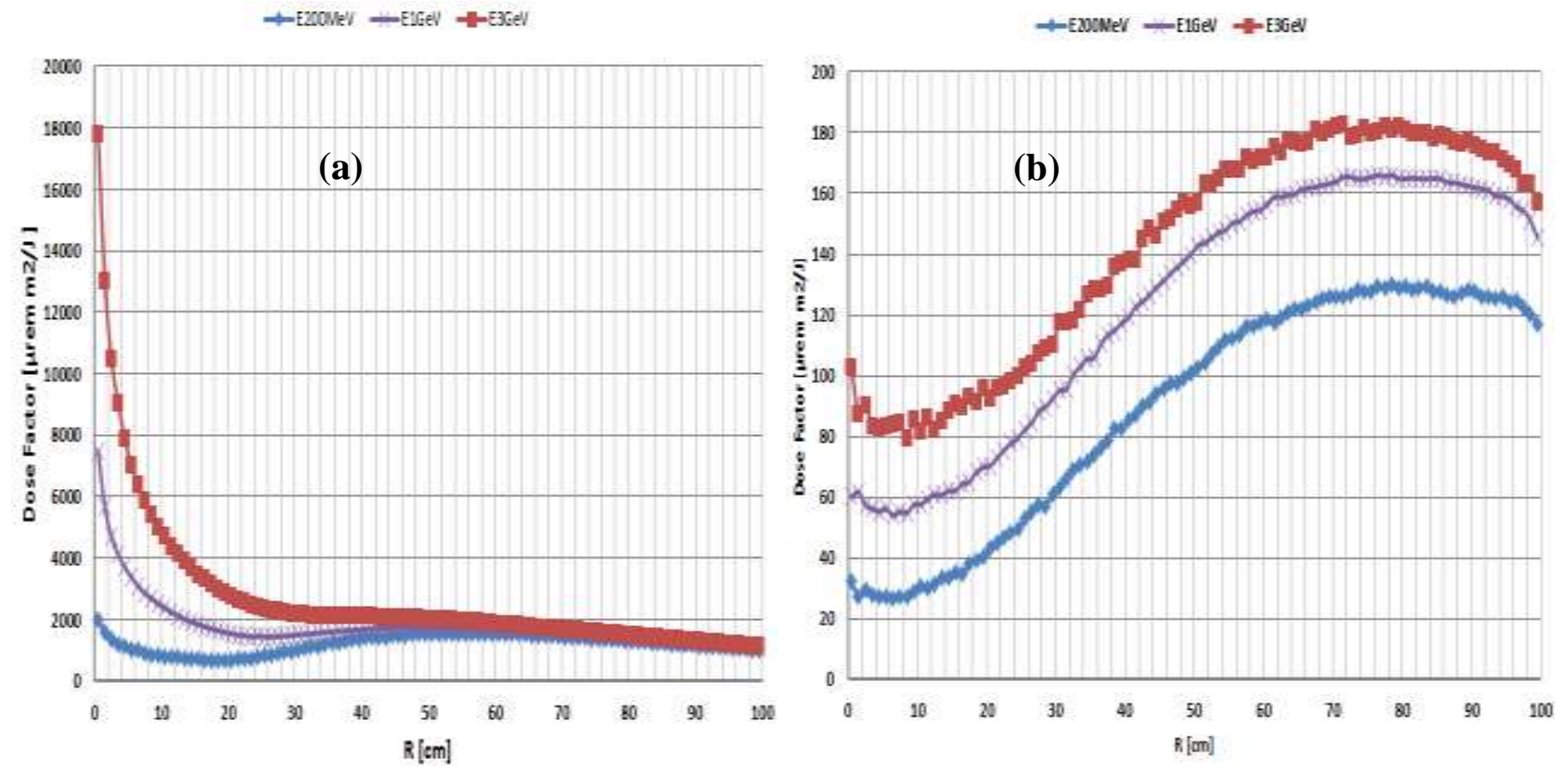

Figure 3 Forward direction normalized ambient dose equivalent versus radial distance from the beam axis at one meter from the target for (a) total and (b) neutron components for the different energies, calculated by FLUKA.
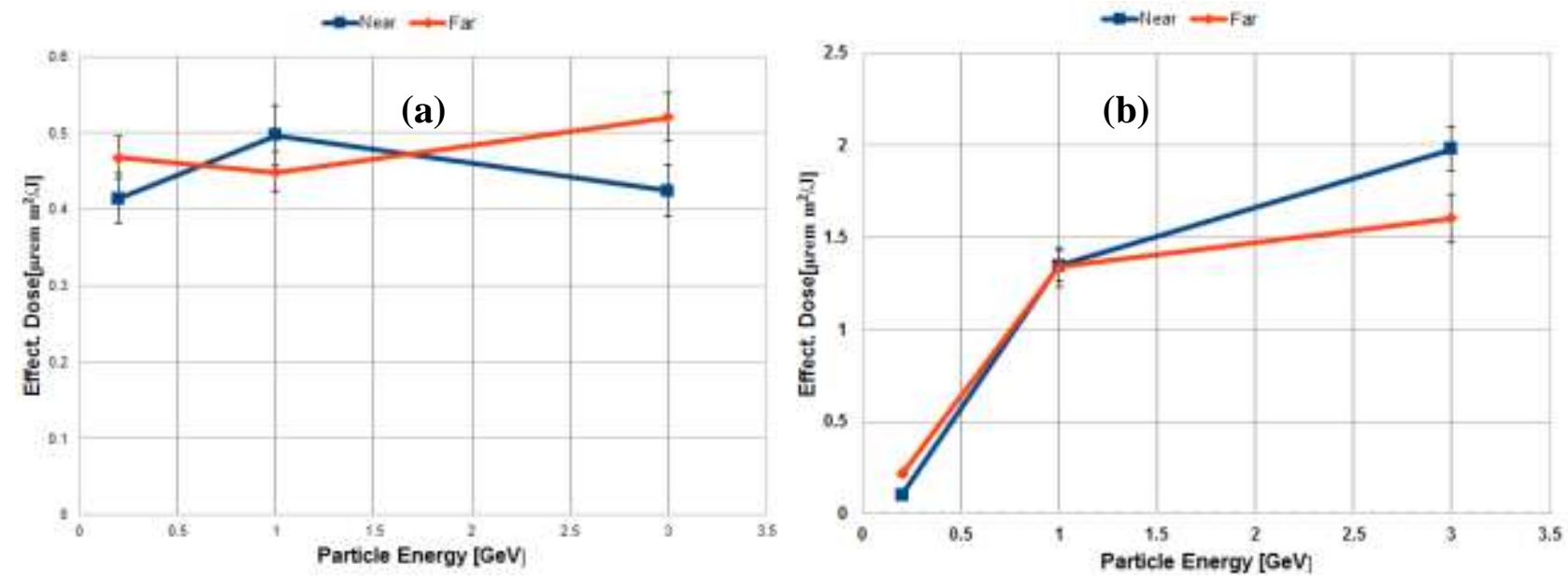

Figure 4 The transverse dose equivalent factors at a distance $\mathrm{R}=2 \mathrm{~m}$ from the target, for the shielded ( $1 \mathrm{~m}$ thick concrete) target beam loss at $0.2,1$ and $3 \mathrm{GeV}$ particle beam energy (a) the gamma and (b) the neutron component for the shield placed Near or Far from the target. 


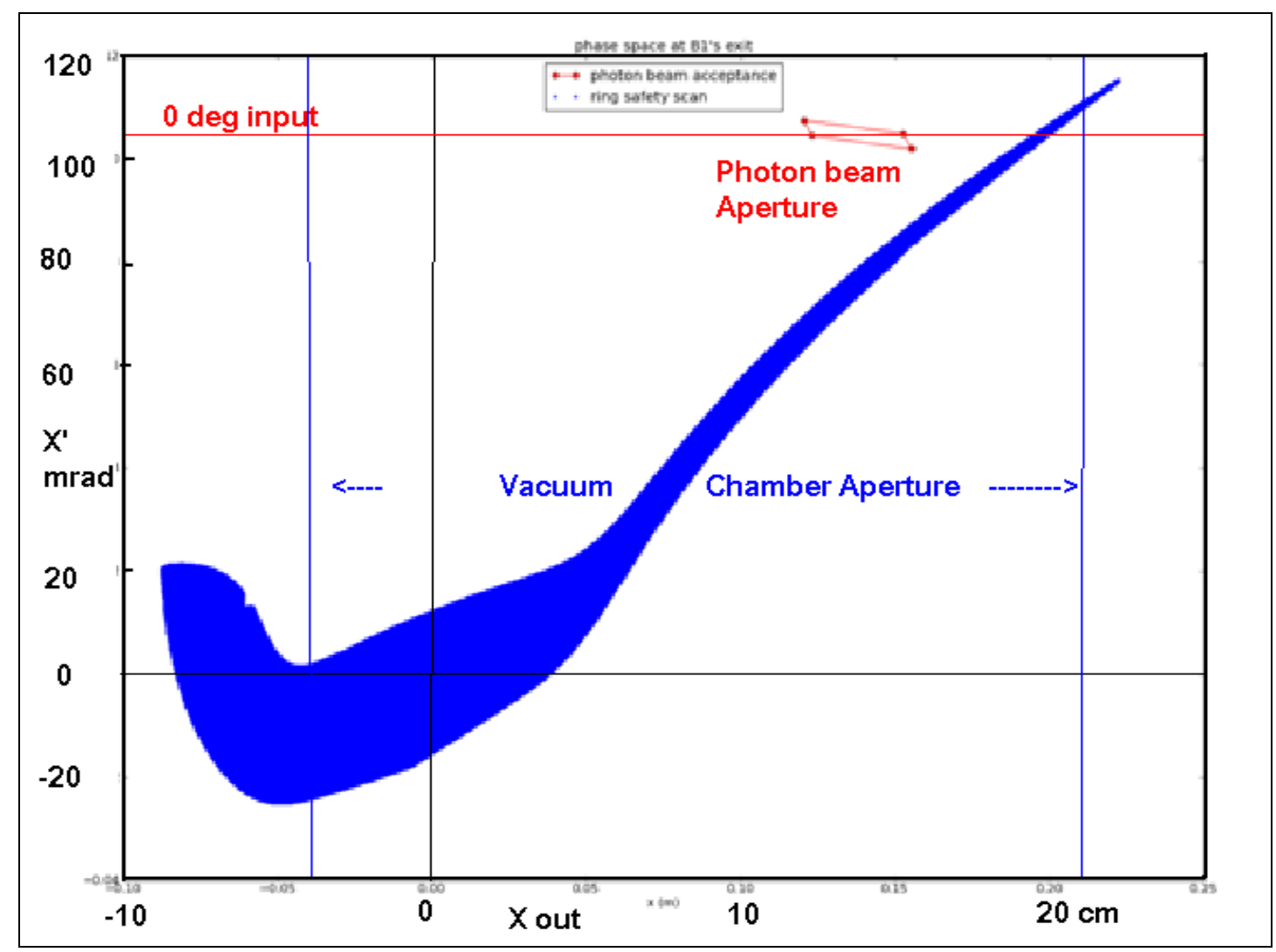

Figure 5 Ensemble of possible miss-steering beam trajectories out of the NSLS-II SR dipole magnet for all possible input beam parameters and magnet setting. The photon beam aperture is shown which has the $0^{\circ}$ bending angle, which is only possible for the first magnet in the series with polarity check assured.

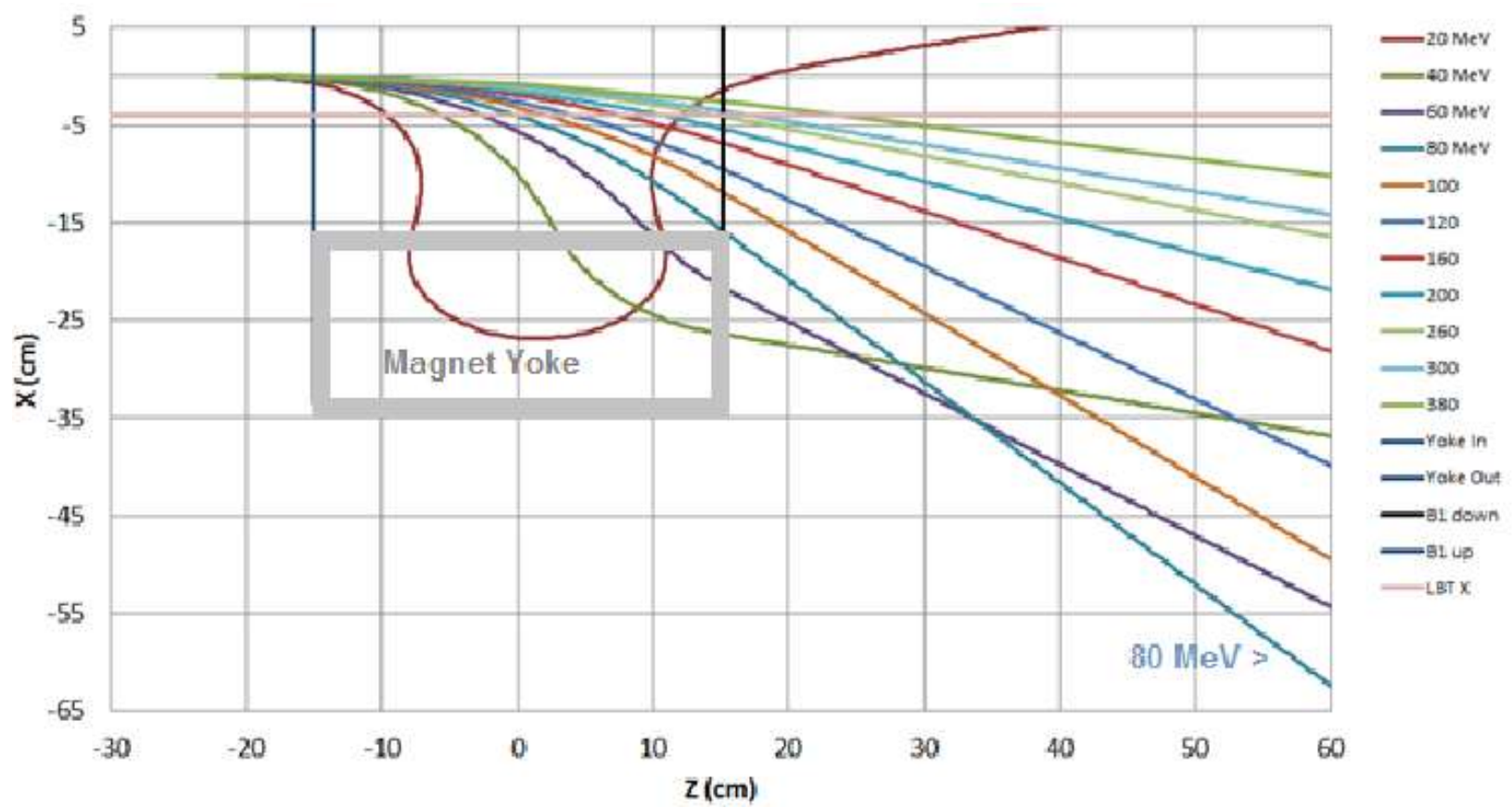

Figure 6 shows the beam trajectories for different beam energies at $82 \%$ of the full field value for the LBT-B1 dipole. 


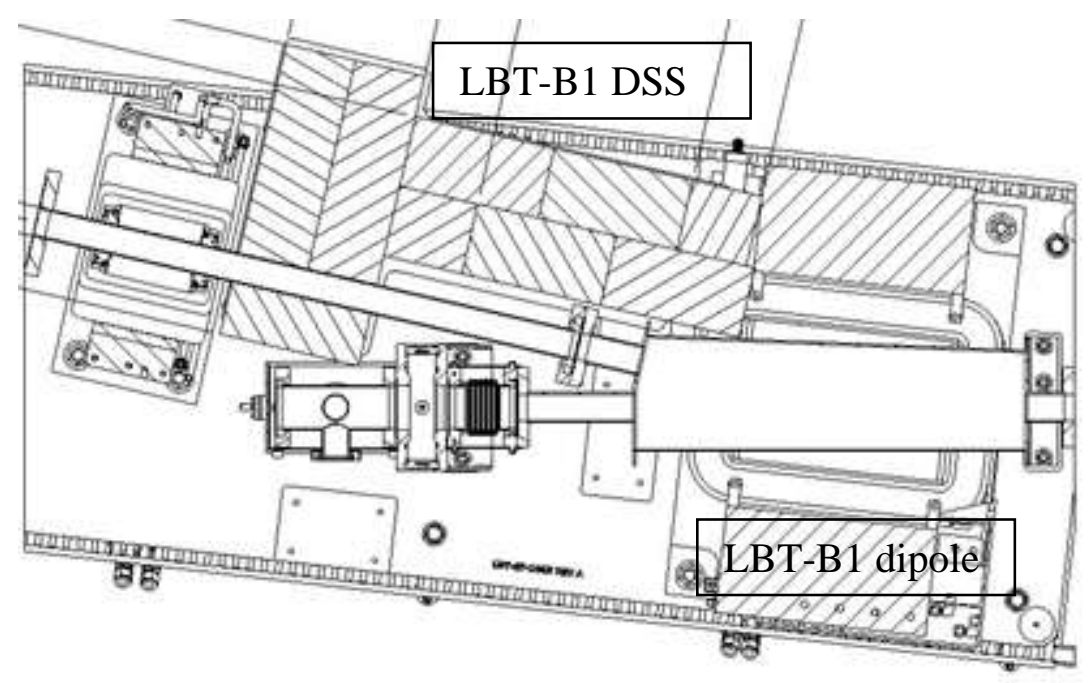

Figure 7 shows the extended LBT-B1 DSS installed between the original (forward) shield and the B1 dipole magnetic yoke.

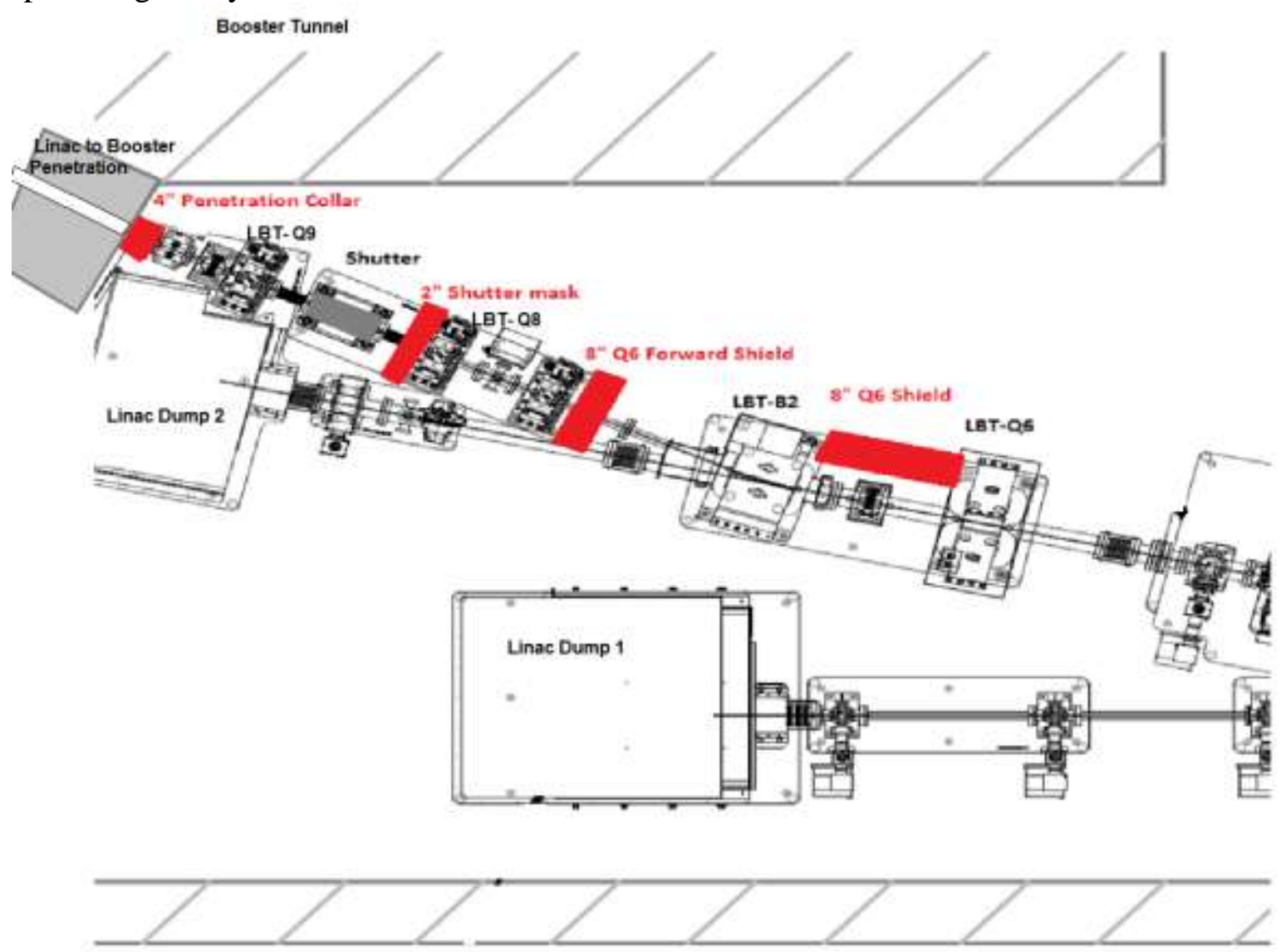

Figure 8 shows the added SSs (shown in red) in the LBT line to shield from quadrupole misssteered beam being transported to Dump2 generating high levels in the Booster Tunnel. 

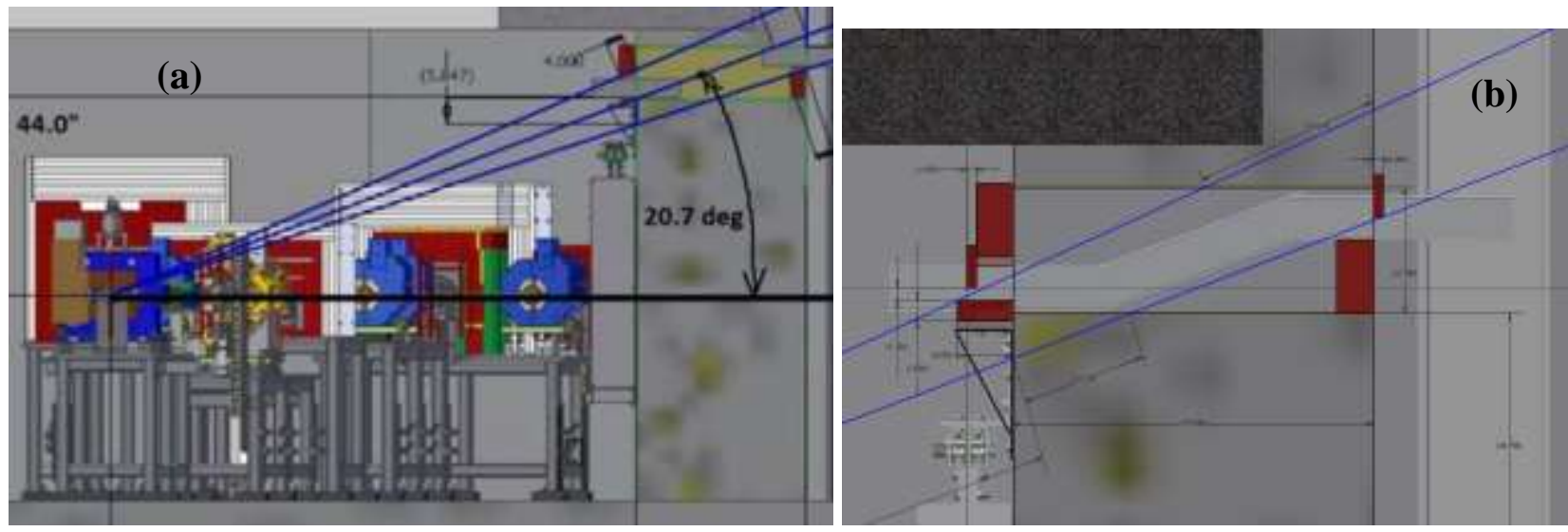

Figure 9 (a) Shows a Linac cable tray penetration with cable tray bend in the same direction as the radiation flow; from low on the left(source) to high on the right (occupied area). (b) Shows the shield wall and supplemental shield bricks (red) to reduce the radiation levels in the occupied area to the right.

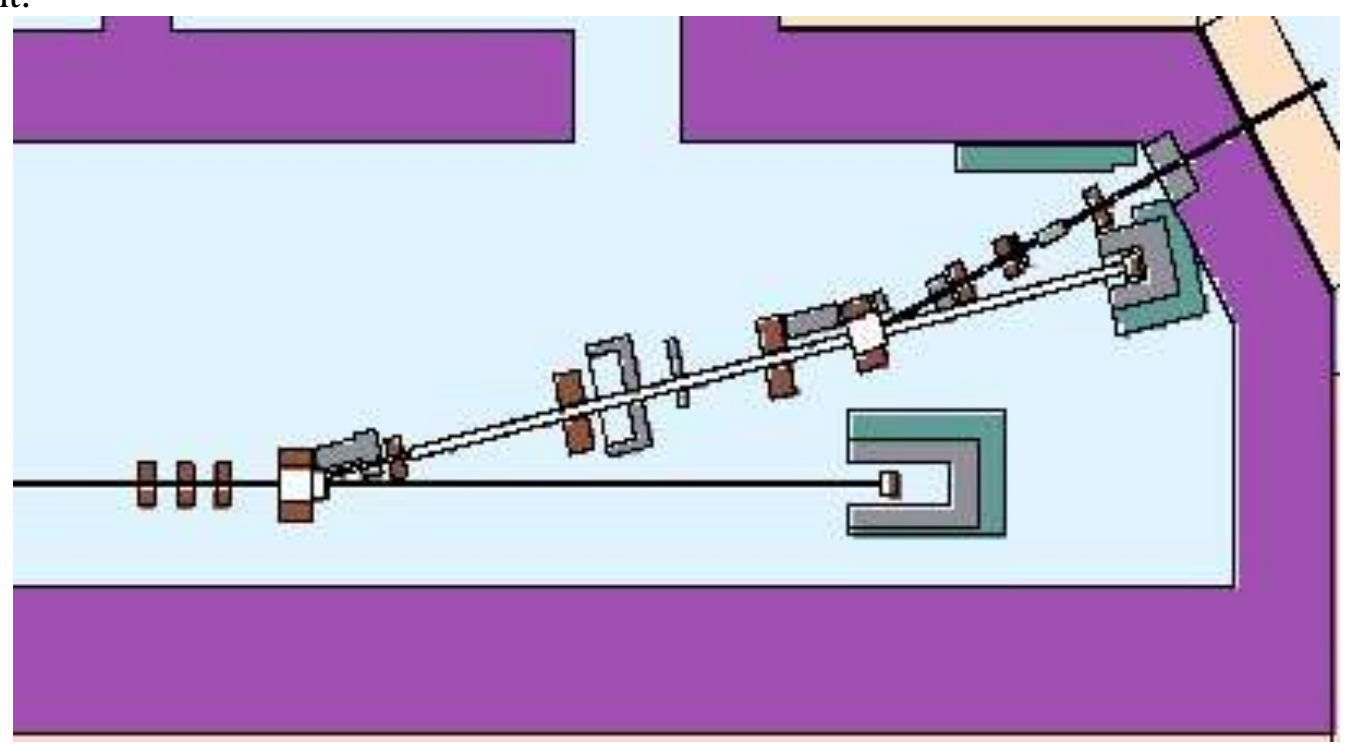

Figure 10 Shows the FLUKA model of the LBT beam line elements (brown) with all specified SSs installed (grey) in the Linac Tunnel bulk shield walls (purple). Both dumps are shown with the polyethylene shields in green. 


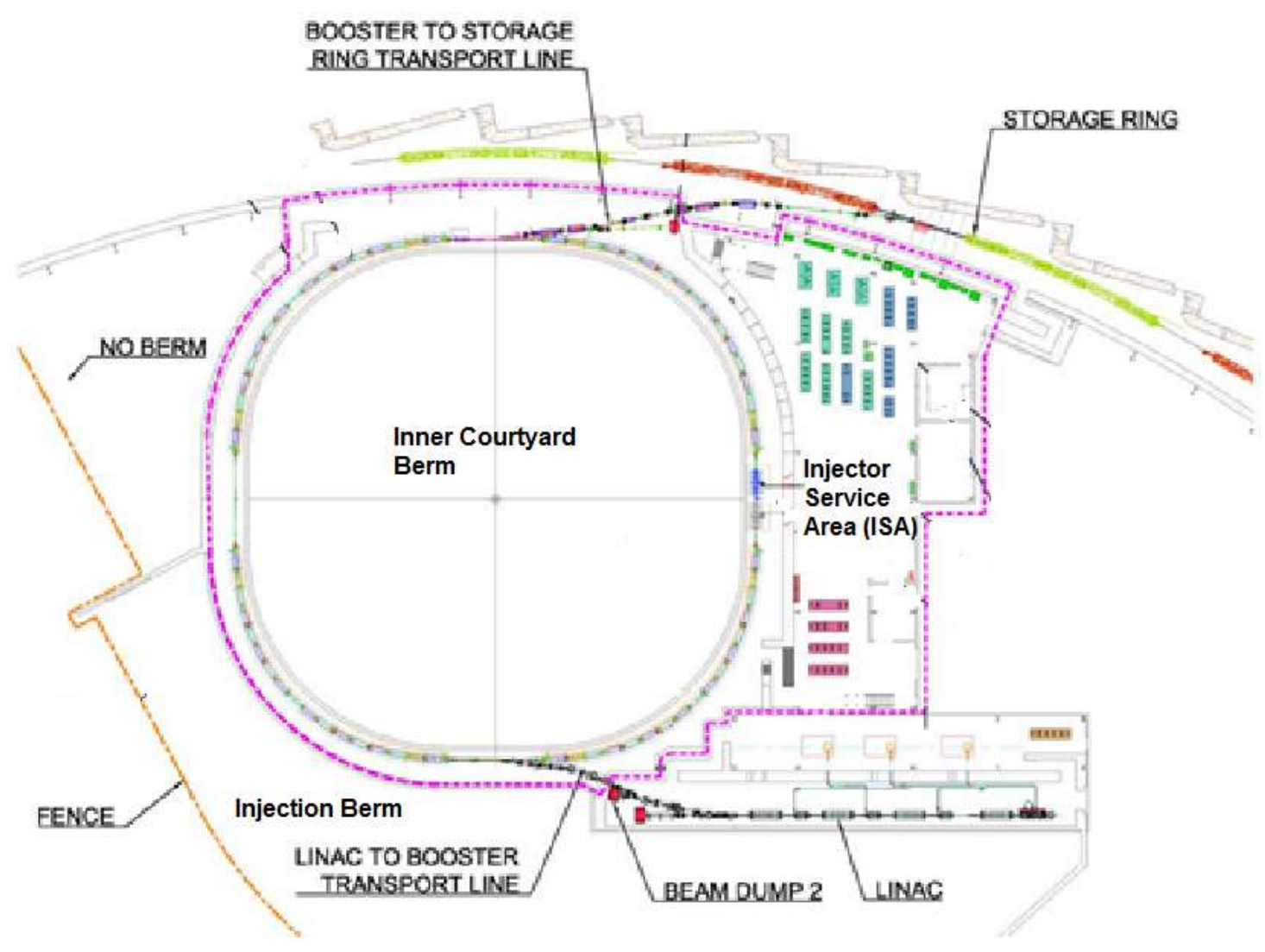

Figure 11(a) Shows the NSLS-II injection complex with the Linac and Booster bulk shielding berm.

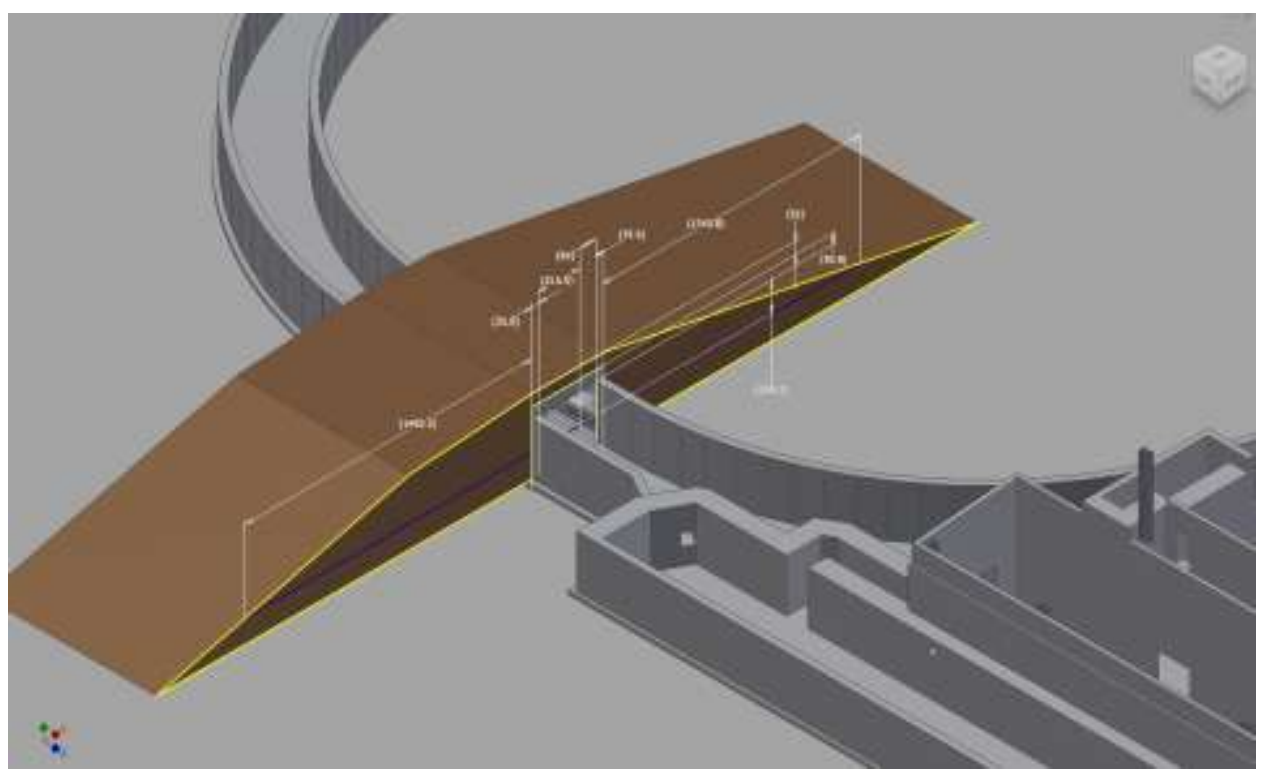

Figure 11(b) Show the cross section of the dirt berm covering the booster and linac tunnels with 15 meters on the beam plane and $>60 \mathrm{~cm}$ dirt above the tunnel. The toe of the berm has interlock fencing to prevent access to the booster and courtyard berms. 


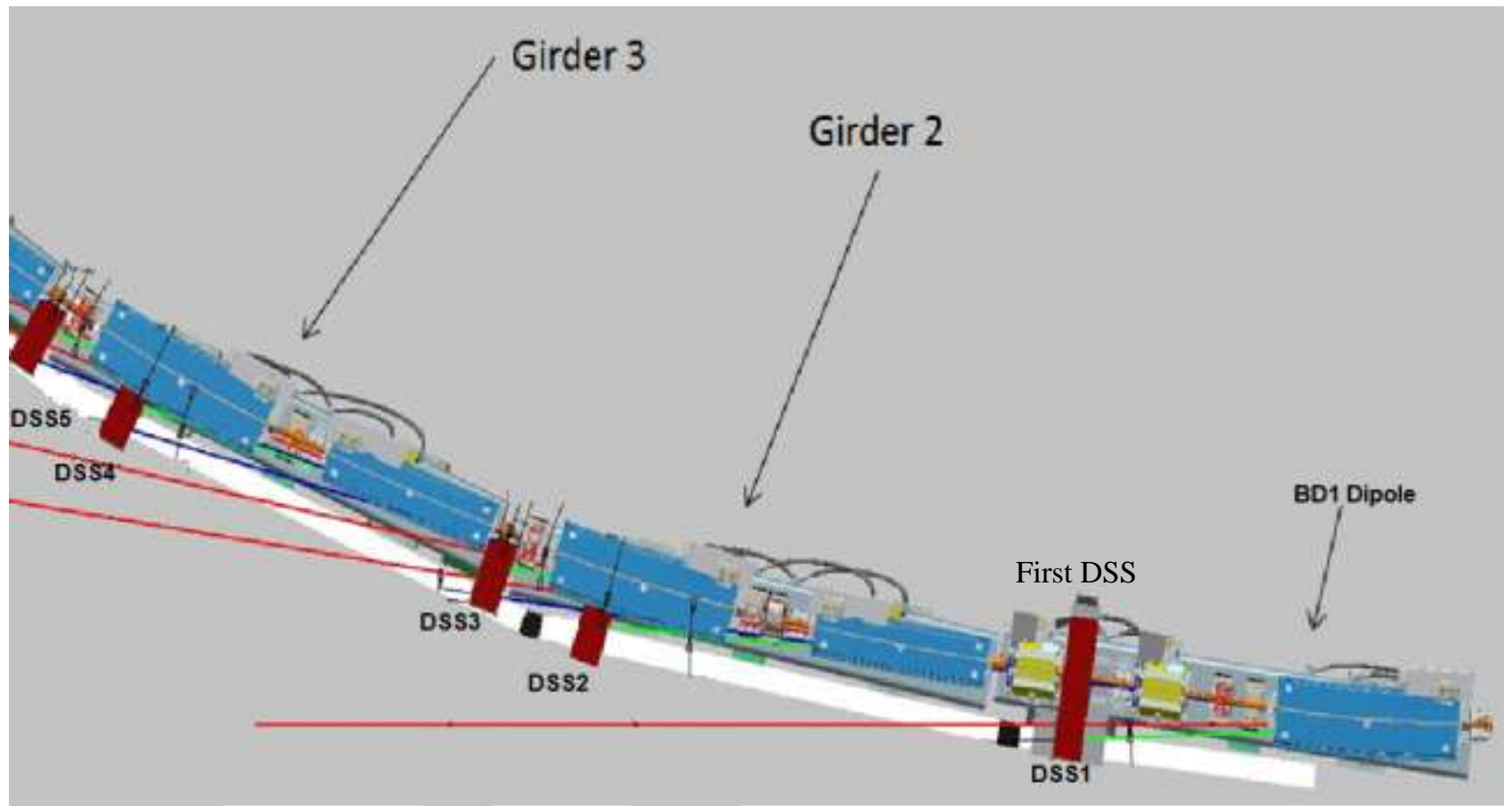

Figure 12 shows the first five DSSs required for the first three dipole girders in the booster arcs.

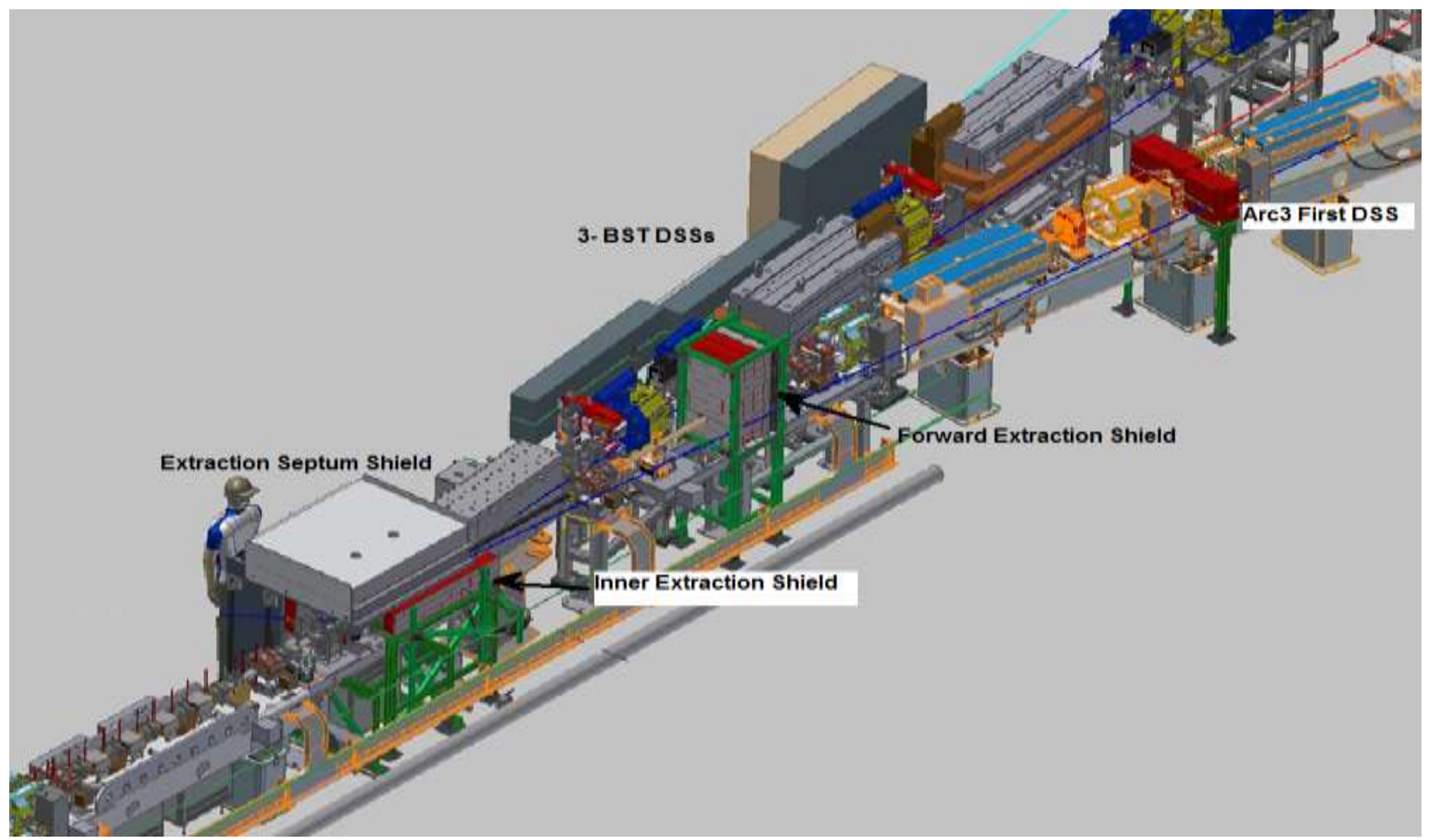

Figure 13 shows the booster extraction septum region and the DSSs installed to intercept the extreme beam loss trajectories for extraction and transport into the BST beam line. The ISA shield wall is toward the right and the SR shield wall is to left and above the figure. 


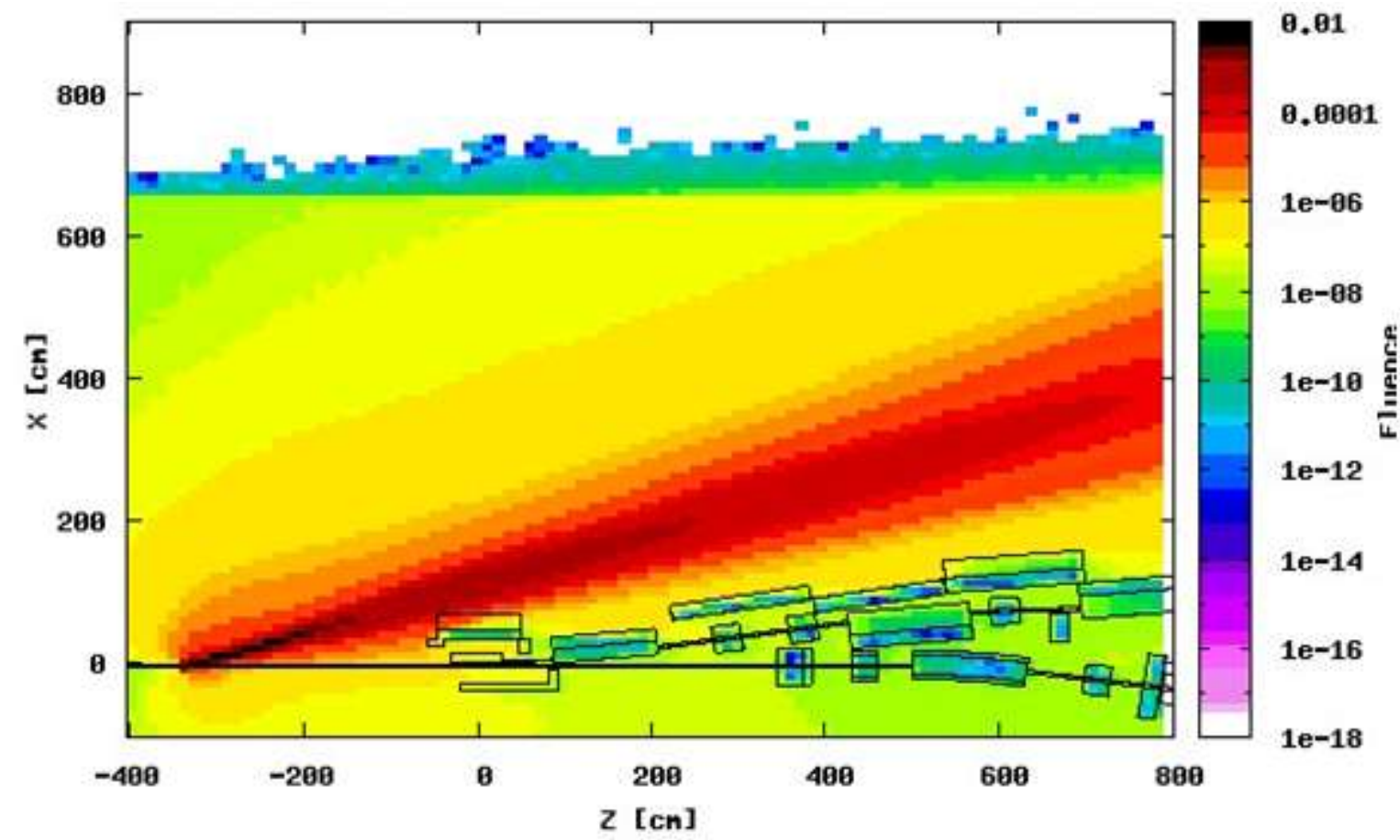

Figure 14 show the electron fluence distribution for $150 \mathrm{MeV}$ booster beam that could be misssteered by the extraction bumps pulsed at the $3 \mathrm{GeV}$ level.

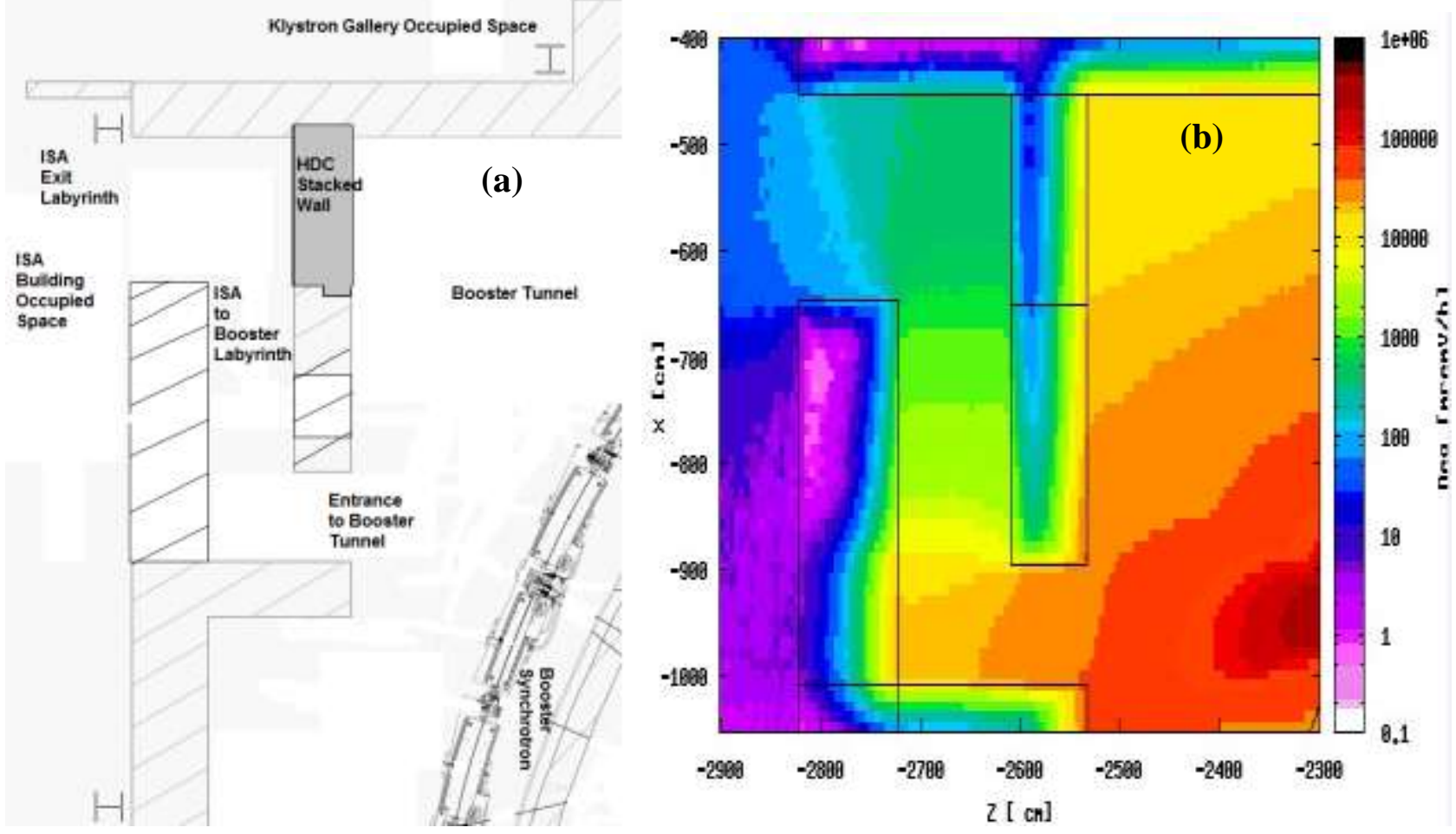

Figure 15 (a) shows the ISA to Booster labyrinth with its stacked (removable) wall for access with fully assembled magnet girders and (b) shows dose distribution in beam plane for $3 \mathrm{GeV}$ beam loss hitting the DSS shield after the third BD magnet in arc4. 

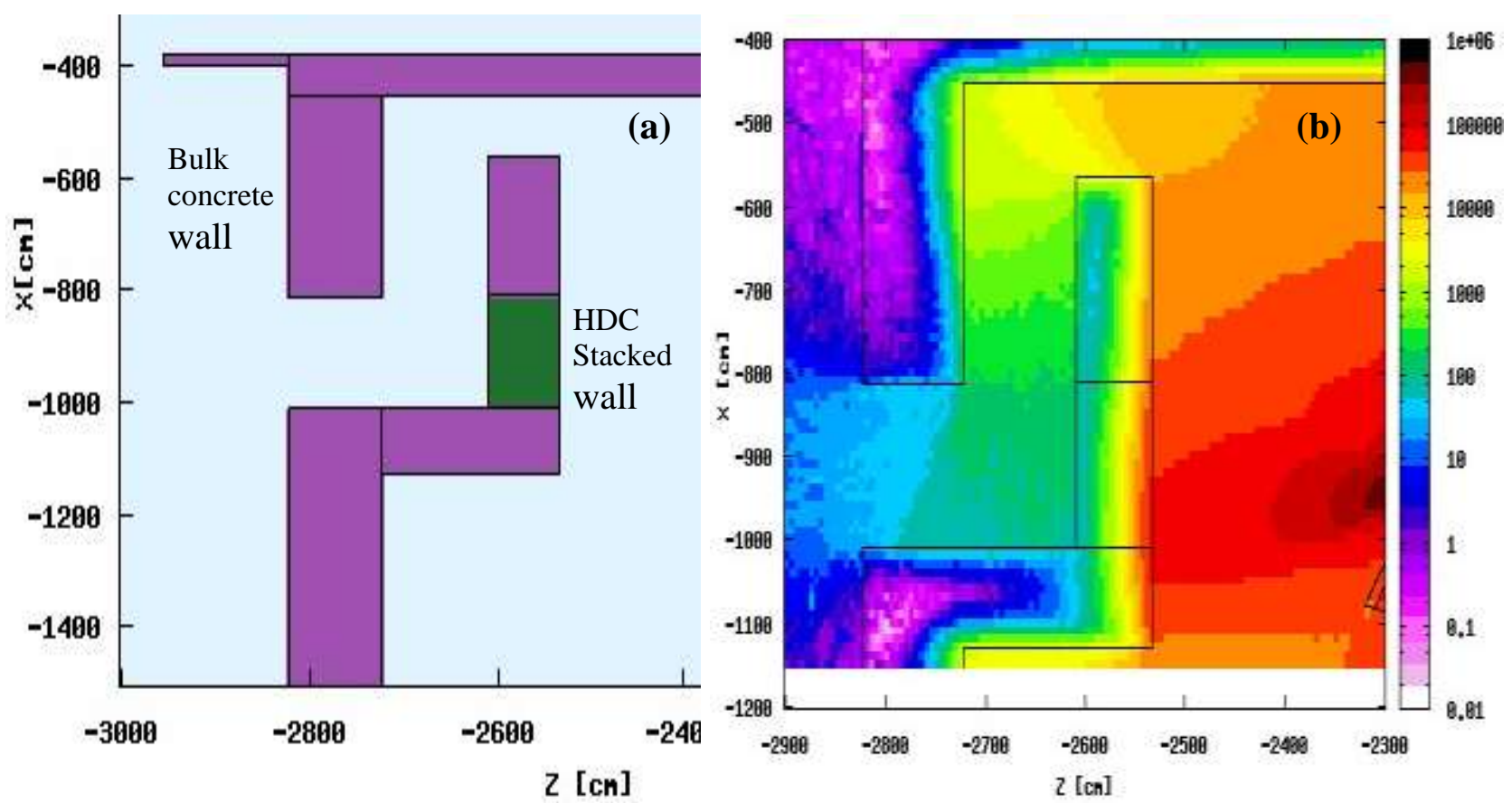

Figure 16 (a) shows the proposed improved labyrinth with its HDC stacked wall and (b) shows dose distribution in beam plane for $3 \mathrm{GeV}$ beam loss hitting the DSS shield after the third BD magnet in arc4, same location as Fig. 15(b).
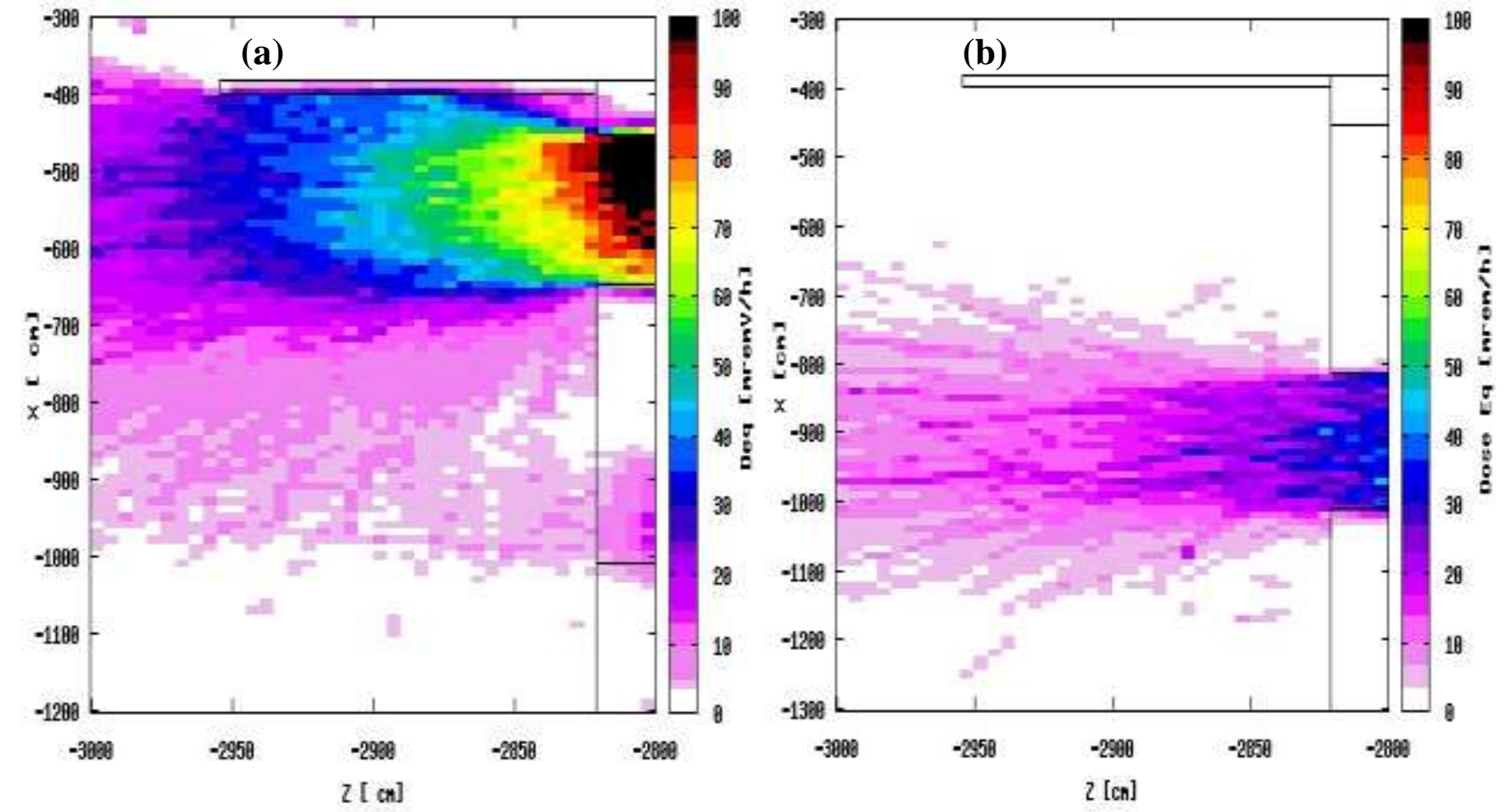

Figure 17 (a) shows beam plane dose distribution exiting the booster labyrinth opening into the ISA for the beam loss shown in Fig. 15(b) and (b) the same dose distribution for the improved labyrinth design as shown in Fig. 16(b). 

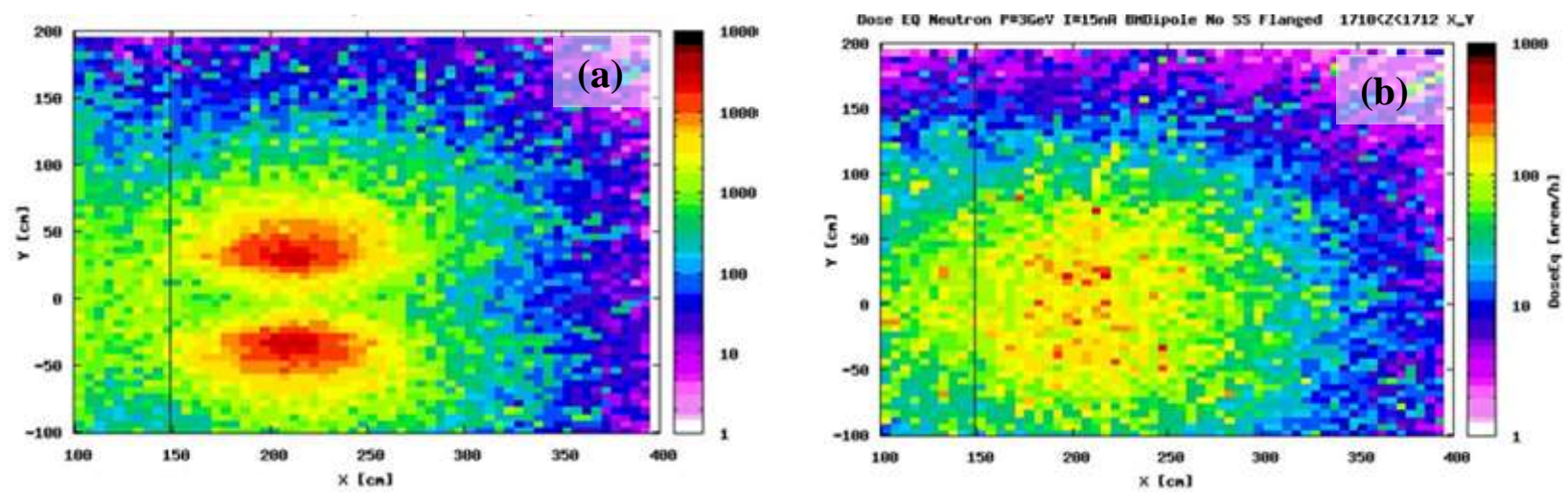

Figure 18 Total dose rate outside the ratchet wall shield for miss-steered $3 \mathrm{GeV}$ injection beam at 15 $\mathrm{nC} / \mathrm{s}$ exiting the SR dipole and hitting the photon absorber and flange $17 \mathrm{~m}$ upstream of the ratchet wall. (a) shows the total dose $<30 \mathrm{rem} / \mathrm{h}(0.3 \mathrm{~Sv} / \mathrm{h})$ and (b) neutron dose rate $<500 \mathrm{mrem} / \mathrm{h}(5$ $\mathrm{mSv} / \mathrm{h})$.

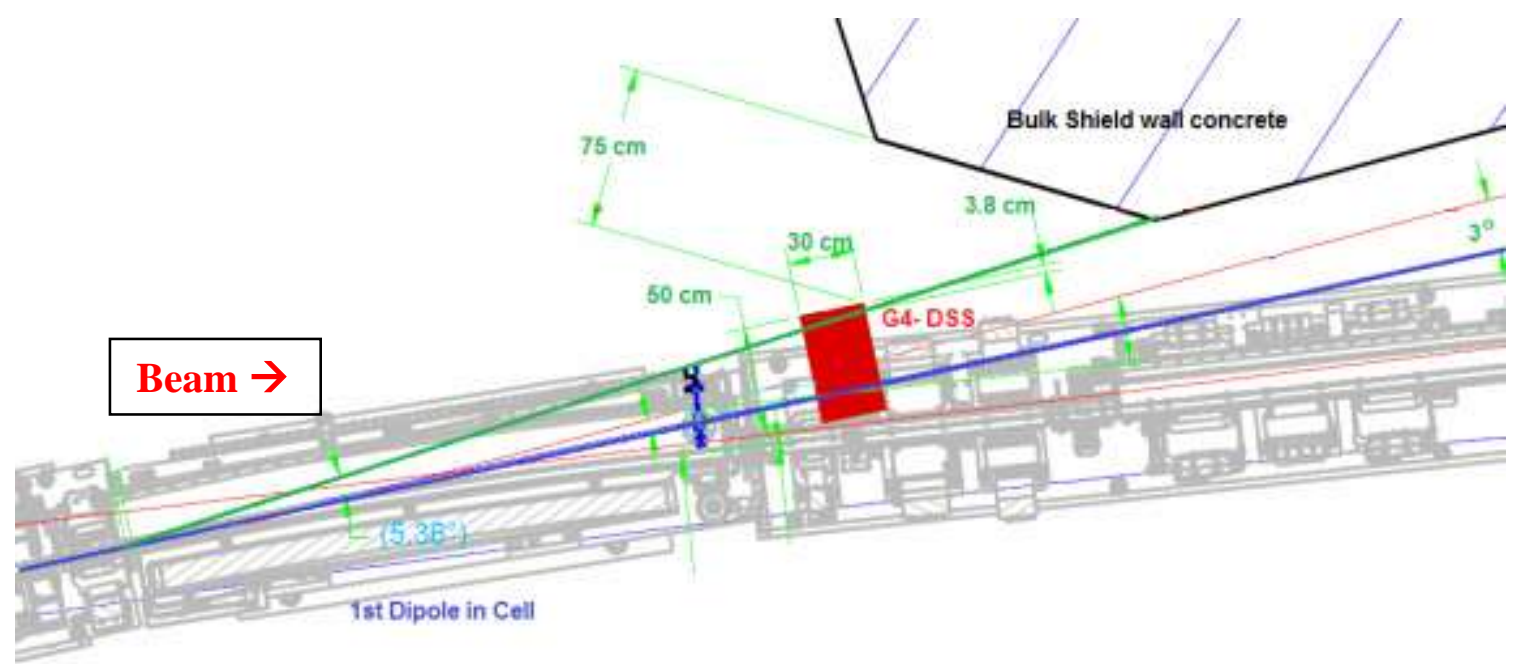

Figure 19 Show the specified first DSSs (G4-DSS) in the SR cell. The outer shield wall limits the angular coverage of this DSS but allowed $>90$ mrad to the limit of the shield wall.

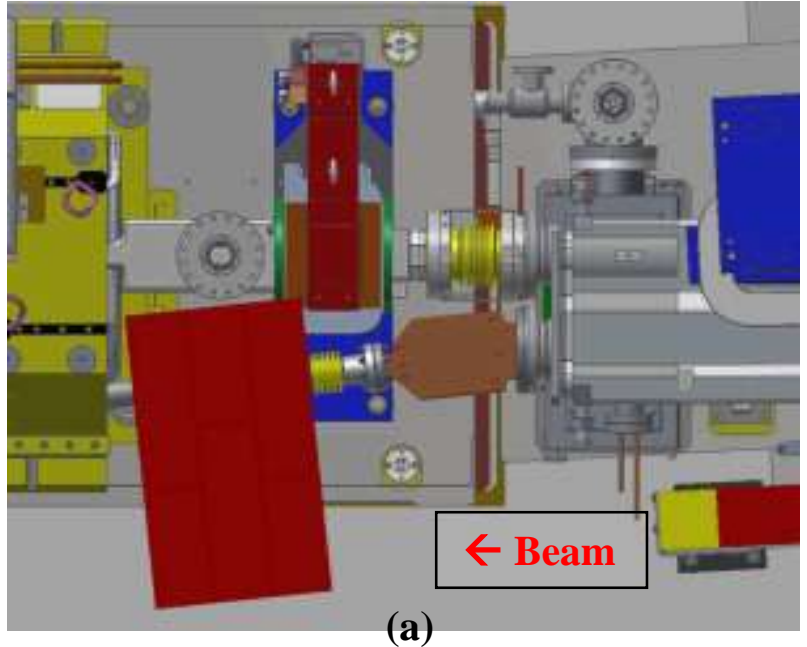

(a)

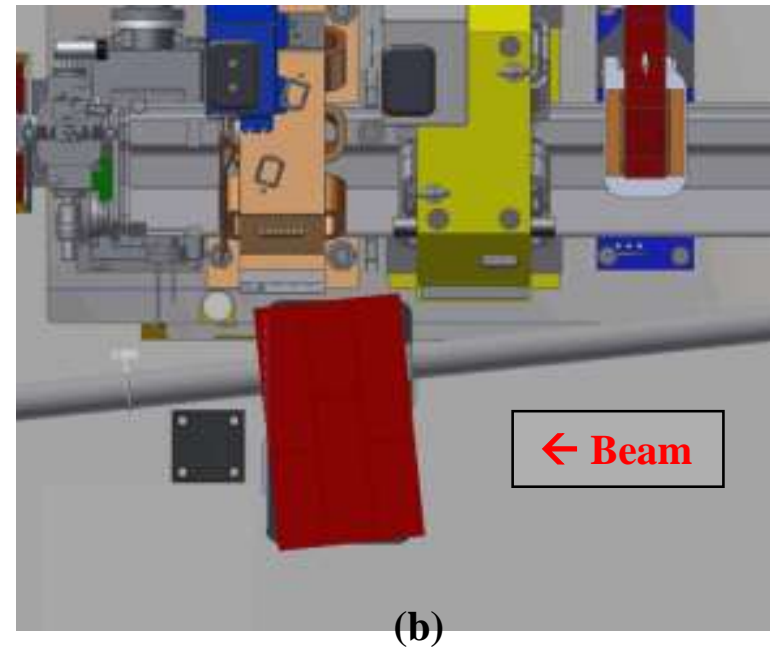

(b)

Figure 20 Show the specified DSS for the SR (a) after the first dipole and (b) after the second dipole. Both show the DSS with an X-Ray beam pipe penetrating the DSS if installed, otherwise the DSS is solid. 

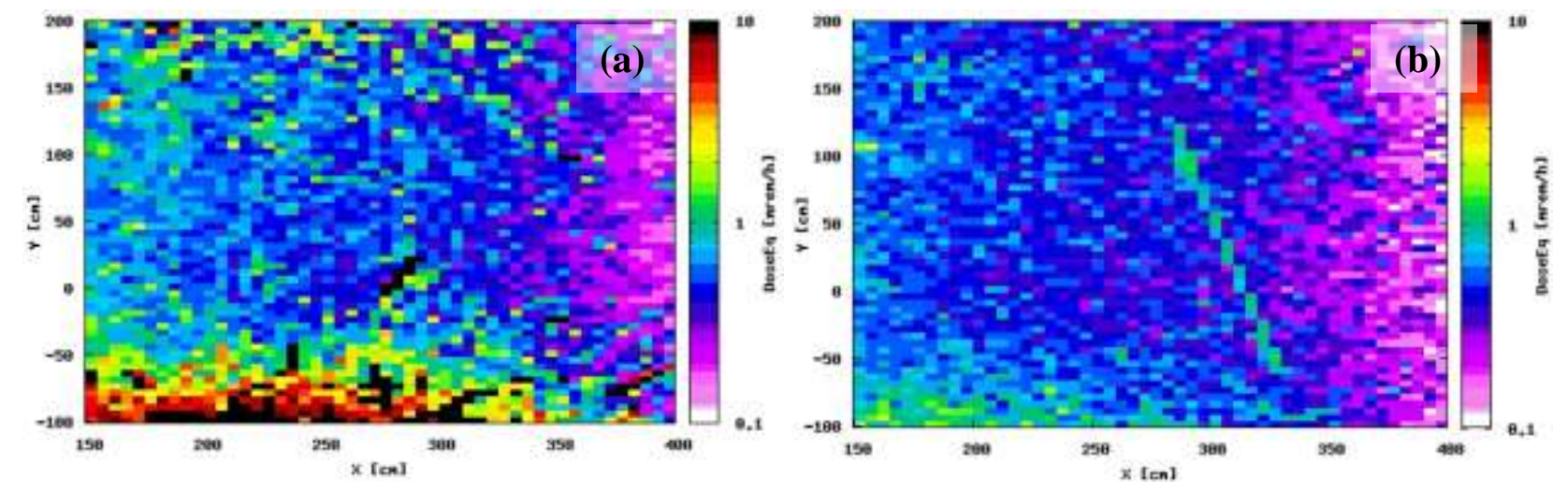

Figure 21 Dose rate outside the ratchet wall shield for same beam loss as in Figure 18 but with the DSS installed after the flange for (a) total dose $<3 \mathrm{mrem} / \mathrm{h}(30 \mu \mathrm{Sv} / \mathrm{h})$ and (b) neutron dose rate $<1$ $\mathrm{mrem} / \mathrm{h}(10 \mu \mathrm{Sv} / \mathrm{h})$.
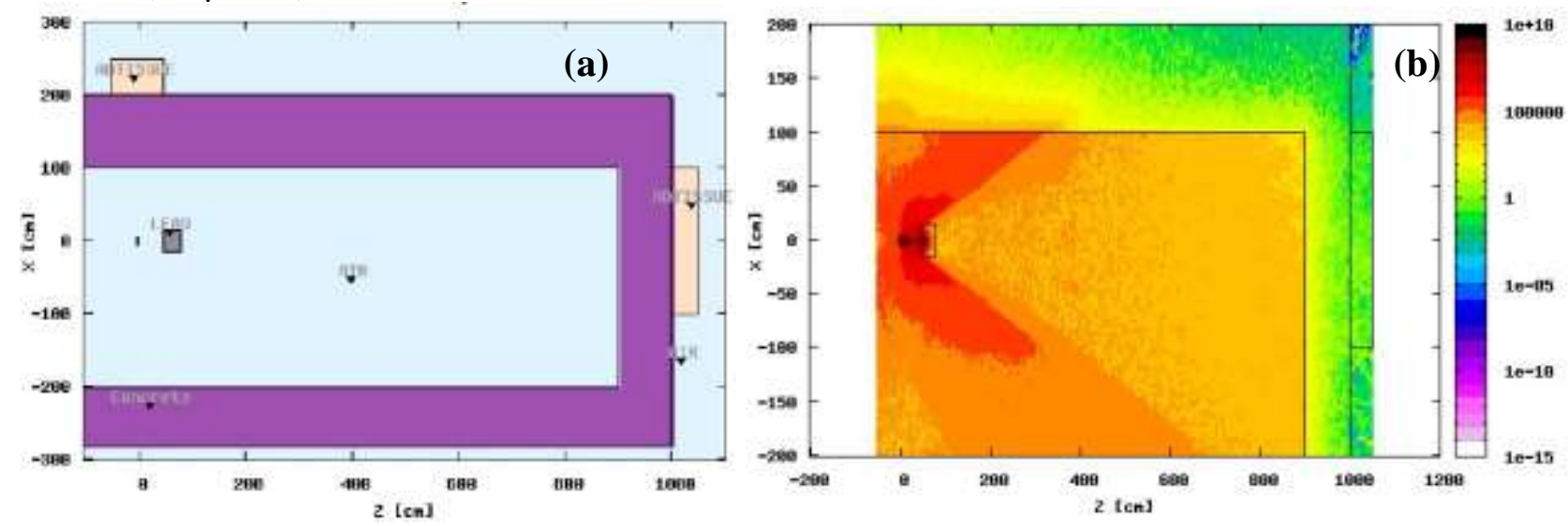

Figure 22 Shows (a) simplified geometry for the thin target forward dose calculation with the $30 \mathrm{~cm}$ thick SS near to the target and (b) the dose distribution in the tunnel for the near SS simulation.

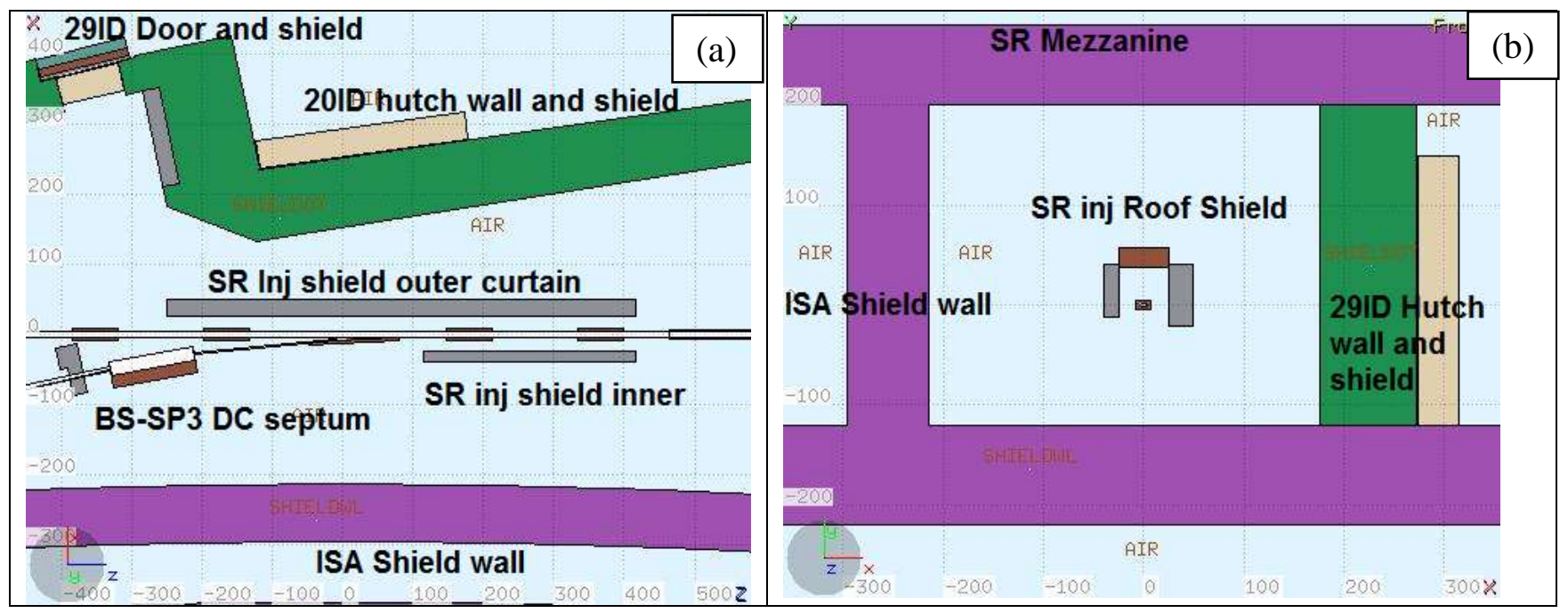

Figure 23 SR injection region showing the SSs at the end of the BST and shields around injection septum and kickers (a) beam plane and (b) vertical plane through the kicker after the pulsed septum. The SR Inj. Roof Shield is made of steel (brown) with sides of Pb (grey). Green walls are the HDC concrete, light tan are the added stacked concrete block walls and purple are normal concrete shield walls. 


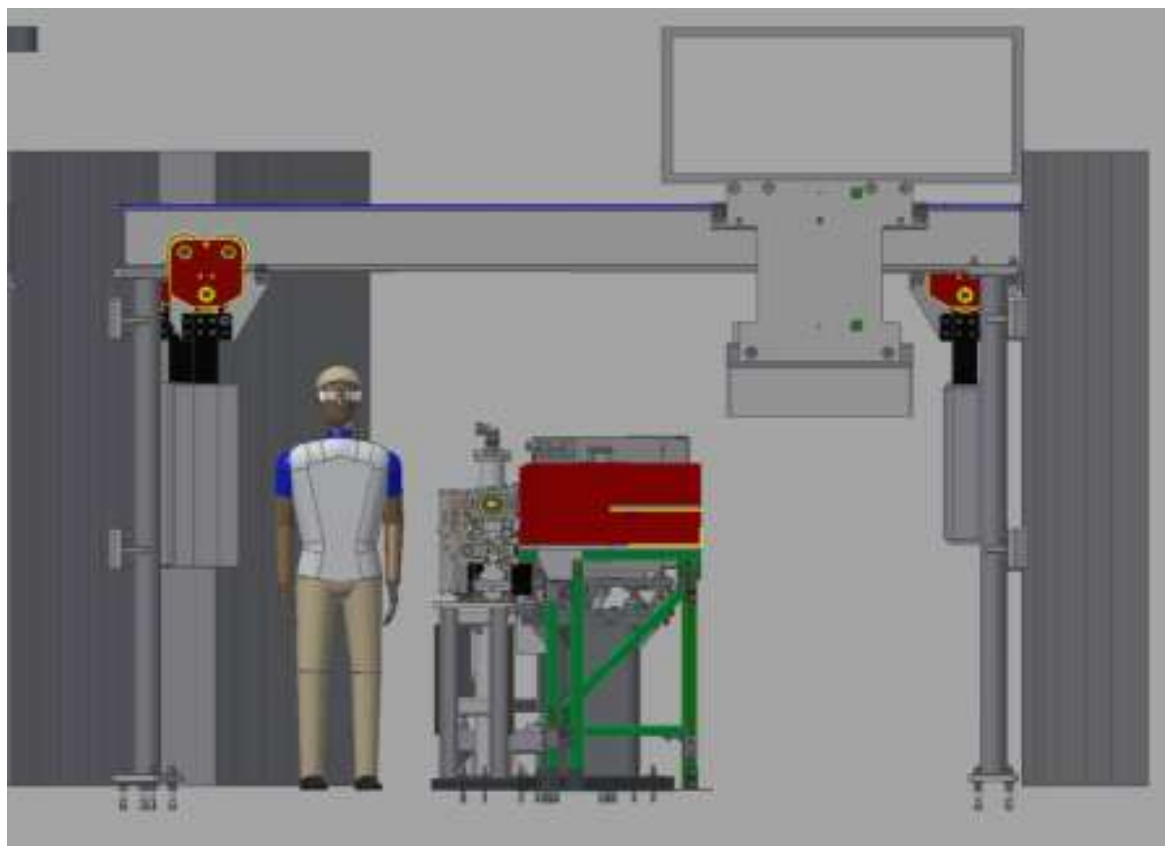

Figure 24 SR injection region overhead shield and side curtains retracted for complete access to injection magnets and their power supplies. Roof shield can retract to either side.
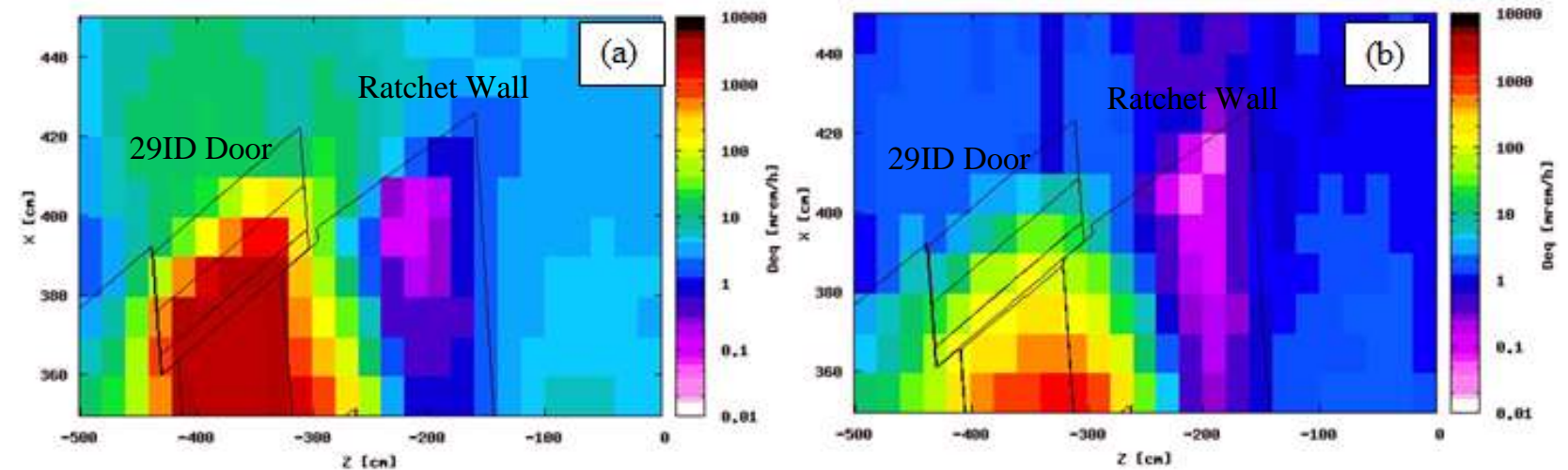

Figure 25 The dose rate at the Cell 29ID door for the DC septum off beam loss condition. The dose rate shown outside the sliding door, (a) with the doorway unfilled and (b) with $50 \mathrm{~cm}$ concrete blocks filling the doorway.

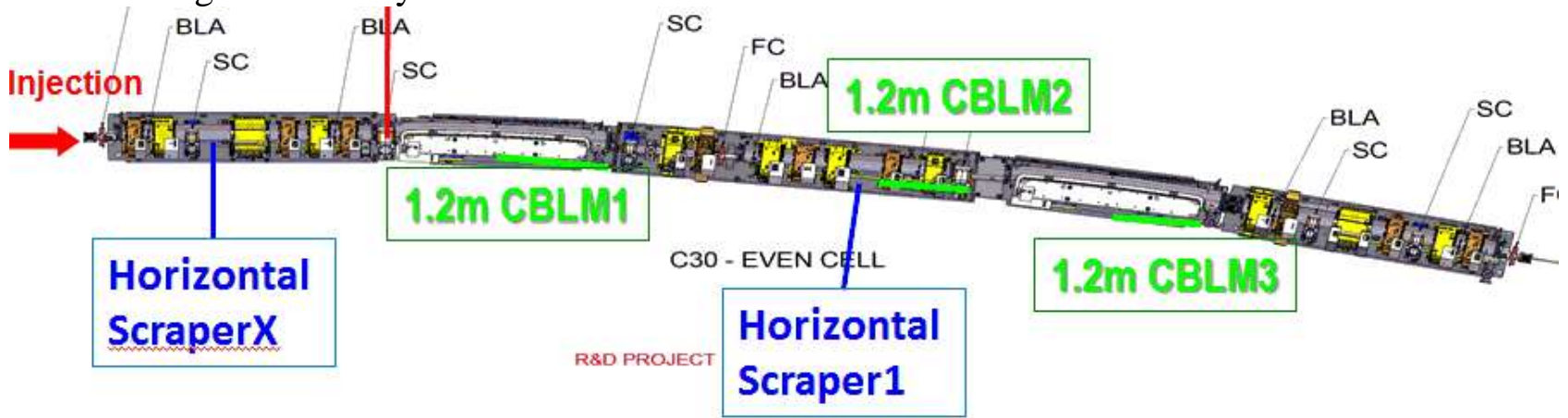

Figure 26 Show the installed scrapers and CBLMs in cell 30 just after injection straight section. All scrapers need to be shielded for high beam loss rates but only Horizontal Scraper 1 and Scraper 2 (located in cell 01) are required for LCM system operations. 


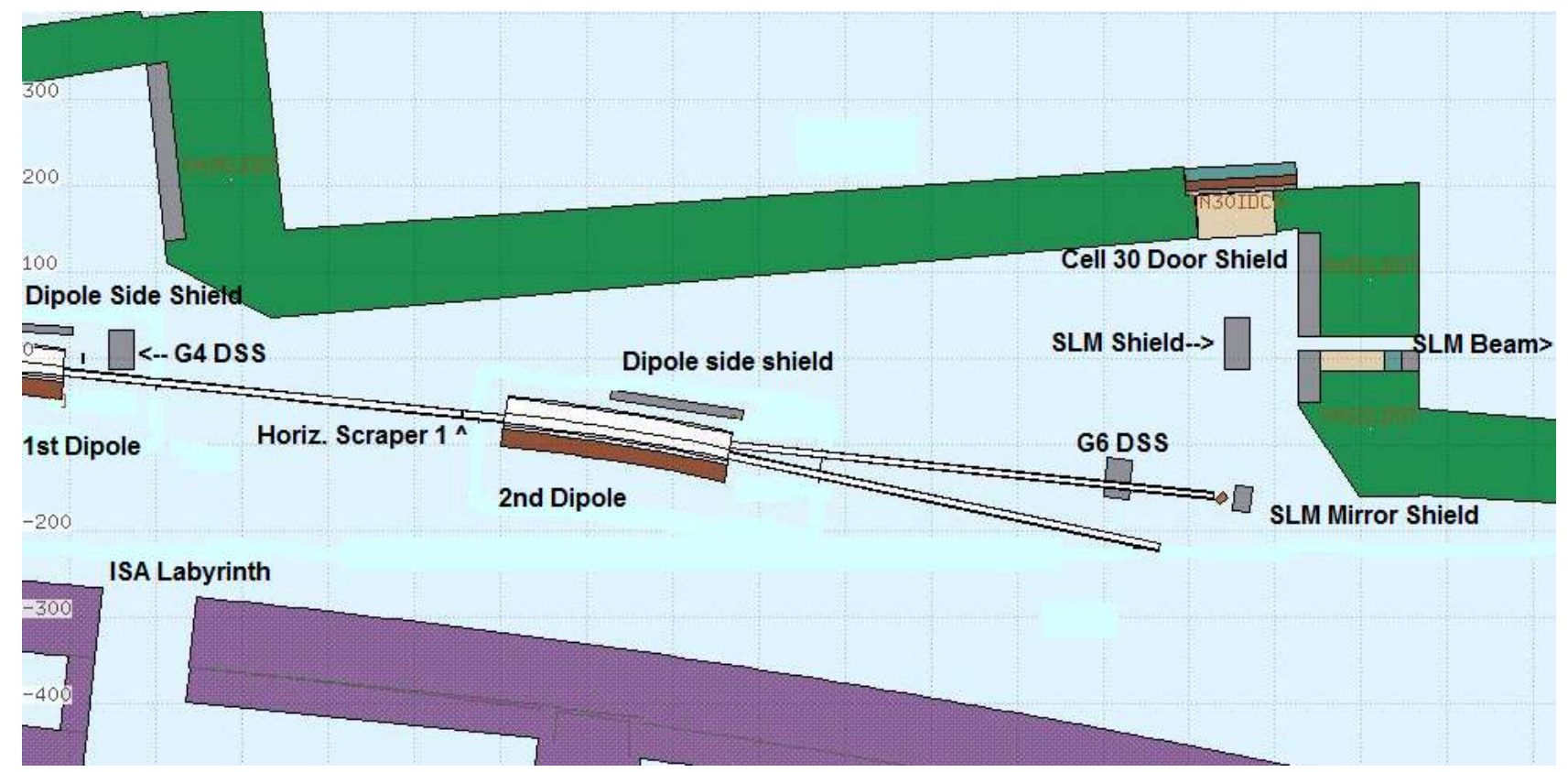

Figure 27 Shows the FLUKA model of the installed SS's specified for the cell 30 second dipole following Horizontal Scraper 1. This dipole is the source of the visible light for the Synchrotron Light Monitor beam line which passes through the ratchet wall. The in air beam pipe after the $90^{\circ}$ mirror isn't shown.
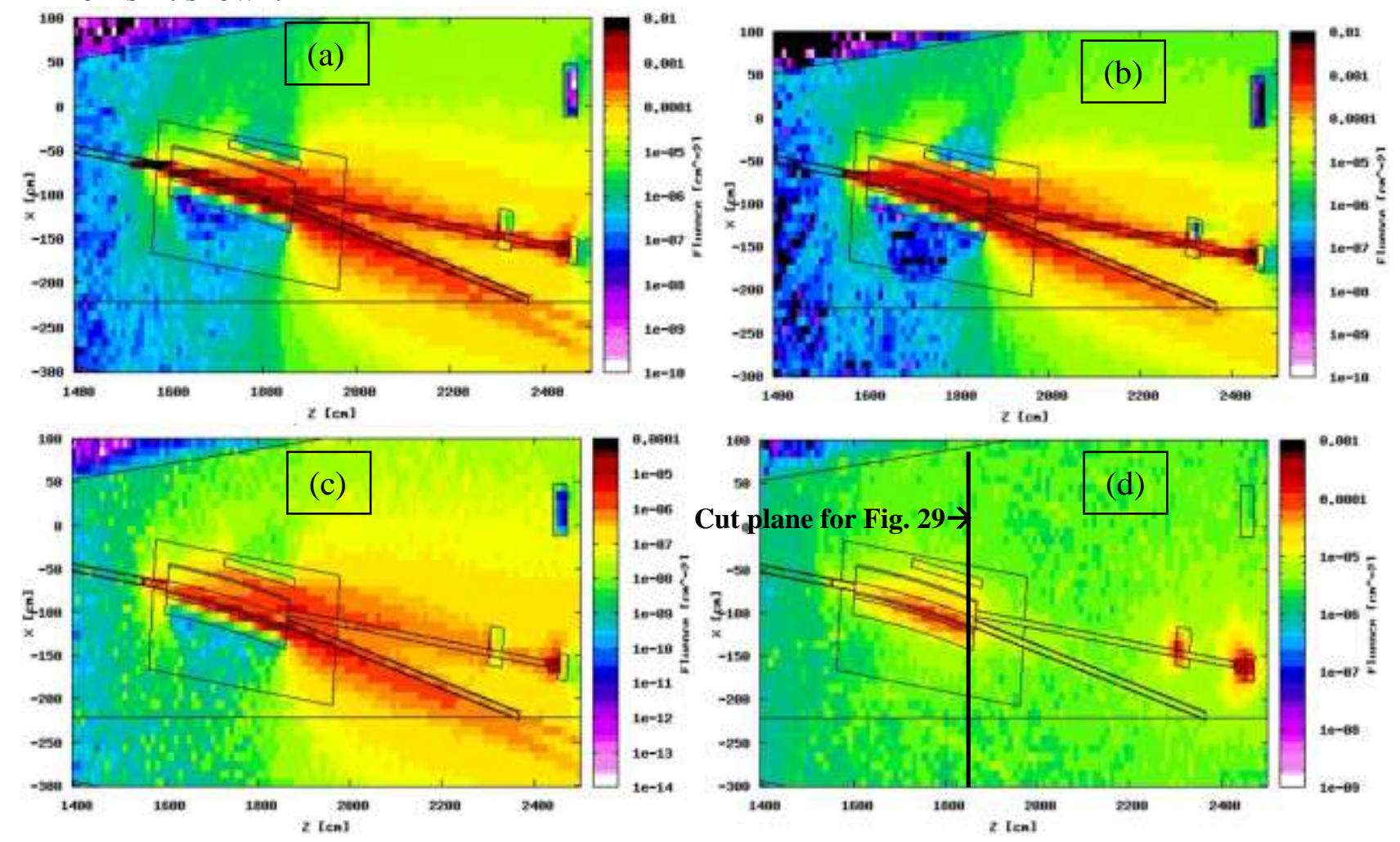

Figure 28 Shows the FLUKA simulation for $3 \mathrm{GeV}$ beam hitting the Scraper with charged particles being bent by the dipole field. The fluence distributions for (a) electrons, (b) positrons, (c) gamma rays and (d) neutrons are shown. 

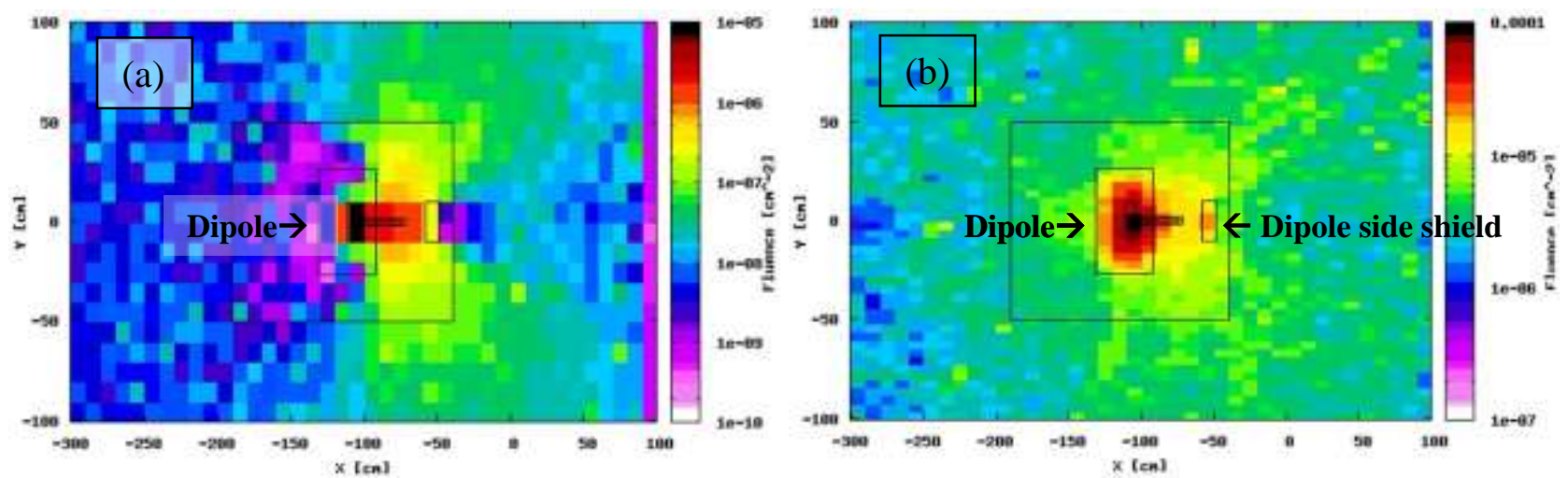

Figure 29 Shows the fluence distribution in the horizontal-vertical cut plane (in Fig. 28) through the dipole for $3 \mathrm{GeV}$ beam hitting the Scraper, as shown in Fig. 28 for (a) gamma rays and (b) neutrons.

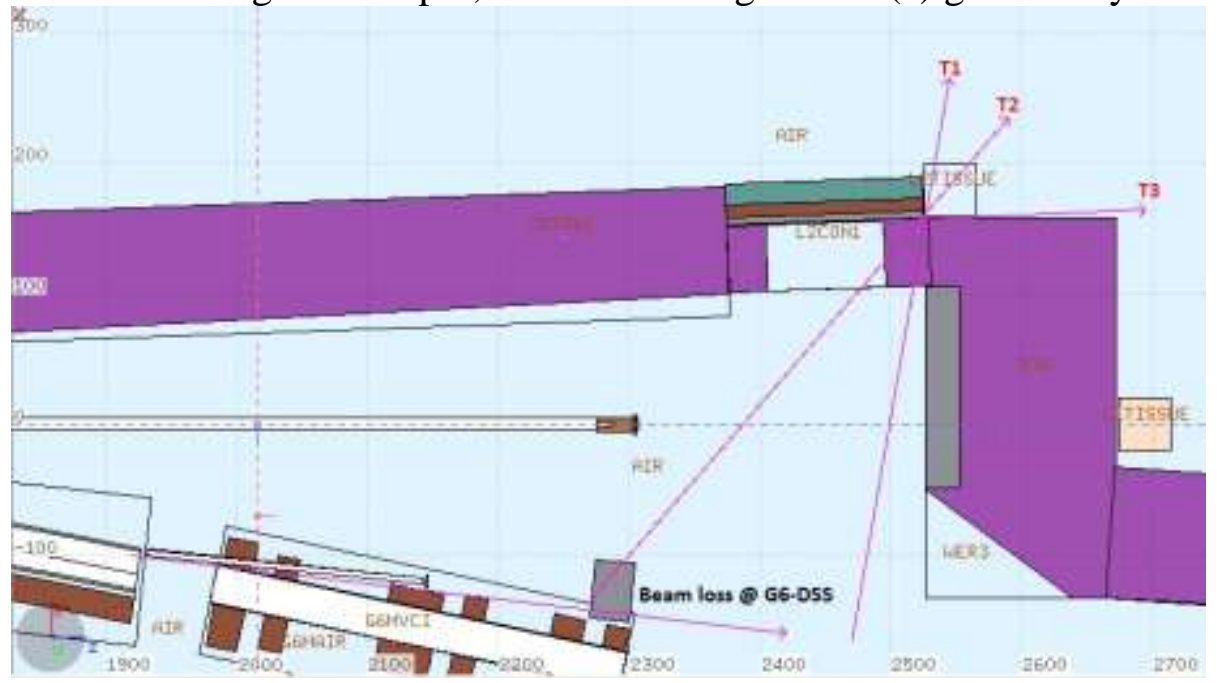

Figure 30 Shows the FLUKA model for a unused damping wiggler beam line which doesn't penetrate the ratchet wall but has a high power photon absorber installed at the end. Shown are three trajectories for radiation by-passing the combined concrete plus door shield attenuation and creating high levels on the experimental floor.

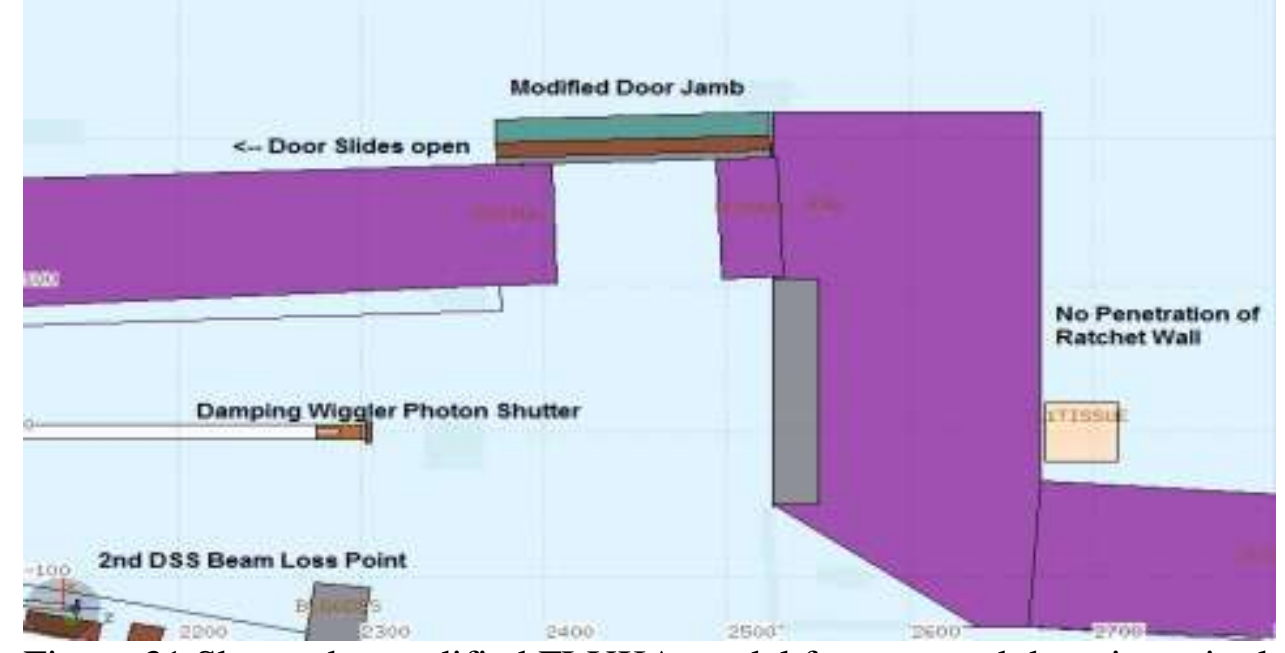

Figure 31 Shows the modified FLUKA model for a unused damping wiggler beam line with a reduced radiation level outside the sliding access door. Note door slide direction is reversed. 

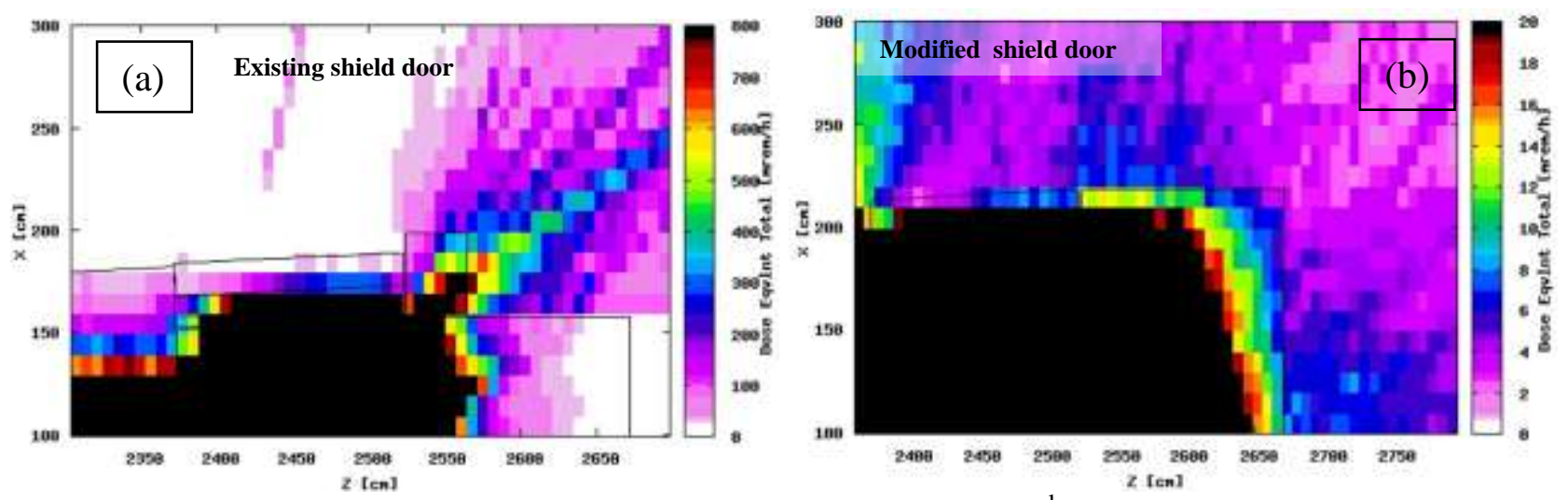

Figure 32 Compares the total dose rate for beam loss hitting the $2^{\text {nd }}$ DSS in the cell for (a) the present door design with full scale dose rate of $800 \mathrm{mrem} / \mathrm{h}(8 \mathrm{mSv} / \mathrm{h})$ and (b) modified shield door design with full scale of $20 \mathrm{mrem} / \mathrm{h}(200 \mu \mathrm{Sv} / \mathrm{h})$. 